\title{
Computation of Hydration Free Energies Using the Multiple Environment Single System Quantum Mechanical/Molecular Mechanical (MESS-QM/MM) Method
}

\author{
Gerhard König ${ }^{1}$, Ye Mei ${ }^{2,3}$, Frank C. Pickard IV ${ }^{1}$, Andrew C. Simmonett ${ }^{1}$, Benjamin T. \\ Miller $^{1}$, John M. Herbert ${ }^{4}$, H. Lee Woodcock ${ }^{5}$, Bernard R. Brooks ${ }^{1}$, and Yihan Shao 6, a) \\ ${ }^{1}$ Laboratory of Computational Biology, National Institutes of Health, National Heart, Lung and \\ Blood Institute, 5635 Fishers Lane, T-900 Suite, Rockville, MD 20852, USA \\ 2State Key Laboratory of Precision Spectroscopy and Department of Physics and Institute of \\ Theoretical and Computational Science, East China Normal University, Shanghai 200062, China \\ ${ }^{3}$ NYU-ECNU Center for Computational Chemistry at NYU Shanghai, Shanghai, China 200062 \\ ${ }^{4}$ Department of Chemistry and Biochemistry, The Ohio State University, Columbus, Ohio 43210, \\ USA
}

${ }^{5}$ Department of Chemistry, University of South Florida, 4202 E. Fowler Avenue, Tampa, FL 33620, USA

${ }^{6} \mathrm{Q}-$ Chem Inc., 6601 Owens Drive, Suite 105, Pleasanton, CA 94588, USA

\begin{abstract}
A recently-developed MESS-E-QM/MM method (multiple-environment single-system quantum mechanical molecular/mechanical calculations with a Roothaan-step extrapolation) is applied to the computation of hydration free energies for the blind SAMPL4 test set and for twelve small molecules. First, free energy simulations are performed with classical molecular mechanics force field using fixed-geometry solute molecules and explicit TIP3P solvent, then the non-BoltzmannBennett method is employed to compute the QM/MM correction (QM/MM-NBB) to the molecular mechanical hydration free energies. For the SAMPL4 set, MESS-E-QM/MM-NBB corrections to the hydration free energy can be obtained 2 or 3 orders of magnitude faster than fully converged QM/MM-NBB corrections, and, on average, the hydration free energies predicted with MESS-EQM/MM-NBB fall within 0.10-0.20 kcal/mol of full-converged QM/MM-NBB results. Out of five density functionals (BLYP, B3LYP, PBE0, M06-2X, and $\omega$ B97X-D), the BLYP functional is found to be most compatible with the TIP3P solvent model and yields the most accurate hydration free energies against experimental values for solute molecules included in this study.
\end{abstract}

\footnotetext{
a) Author to whom correspondence should be addressed; yihan@ q-chem.com. SUPPORTING INFORMATION

Implicit solvation free energies, gas-phase dipole moments, HOMO-LUMO gaps and optimized $\lambda$ value for the molecules studied in this work are available in the Supporting Information. The average changes in QM/MM permanent electrostatic energy and in QM/MM polarization energy with density functionals are also included for the twelve small molecules. Also included are the QM/MM-NBB-corrected hydration free energies with different sampling frequencies. This information is available free of charge via the Internet at http://pubs.acs.org.
} 


\section{Graphical Abstract}

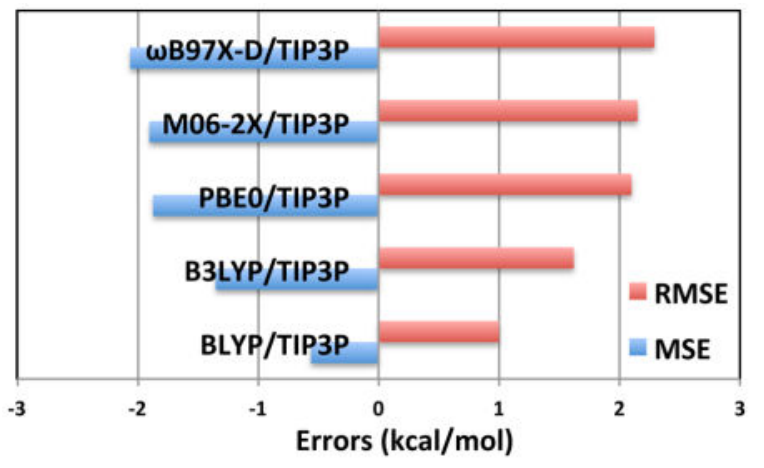

\section{INTRODUCTION}

The prediction of solvation free energy, which is the energy required to transfer a solute molecule from vacuum to a solvent environment, is of both scientific and methodological interest. Scientifically, an accurate prediction of solvation free energy can aid many areas of basic and applied research, such as (a) solvent selection in inorganic/organic synthesis; (b) drug solubility prediction, which is often carried out via a two-step process: first from crystal to vacuum, and then from vacuum to solvent; (c) ligand binding free energy prediction, where a ligand is transferred from a pure solvent environment to a macromolecular environment; and (d) pKa predictions. Methodologically, the prediction of solvation free energies has been an important way to assess the performance of various free energy methods ${ }^{1-9}$ thanks to the availability of reliable experimental solvation free energies for thousands of solute/solvent combinations.

In particular, the statistical assessment of modeling of proteins and ligands (SAMPL) challenges, ${ }^{5-9}$ which started in 2008 , have provided a general picture about the accuracy of various computational methods for predicting solvation free energies. In the most recent SAMPL4 challenge, for example, it was observed that ${ }^{9}$ "many methods are apparently converging on robust, predictive protocols with RMS errors under $1.5 \mathrm{kcal} / \mathrm{mol}$ ". Methods used in the challenge included: (a) knowledge-based models; (b) molecular mechanics (MM) simulations with implicit PB/SA solvent; (c) ab initio quantum mechanics (QM) calculations in implicit solvent; (d) MM simulations with explicit solvent, with and without hybrid quantum mechanical molecular mechanics (QM/MM) corrections, etc.

In this work, we shall focus mainly on the last category of methods, i.e. hydration free energy calculations with explicit solvent, with special attention paid to the accuracy and efficiency of the QM/MM correction to MM hydration free energies. In the computation of MM hydration free energies, the accuracy is controlled by three obvious factors: (a) the MM force field; (b) the free energy simulation method; and (c) the length of each trajectory in the free energy simulation. For the QM/MM correction, one takes the ensemble as generated from the MM simulation, and uses the QM/MM energy to reweight configurations selected at regular intervals, which should in principle lead to an improved value for the hydration free energy. Needless to say, the quality of QM/MM corrections depends on the underlying 
QM/MM energy function, which involves: (a) the QM method in use; (b) the MM charges that polarize the QM wavefunction; and (c) the parameterization of the van der Waals (vdW) interactions between $\mathrm{QM}$ and $\mathrm{MM}$ atoms. In addition, a $\mathrm{QM} / \mathrm{MM}$ correction can improve the accuracy of MM hydration free energy only if the MM potential energy surface (PES) in use significantly overlaps with the $\mathrm{QM} / \mathrm{MM} \mathrm{PES},{ }^{10,11}$ i.e. all essential configurations are already sampled in the MM ensemble which requires an agreement of the bonded terms.

Given all of these factors, it is very encouraging that, in two explicit solvent simulations in the SAMPL4 challenge, MM results were improved upon with a QM/MM correction. Genheden et al ${ }^{12}$ performed all-atom Monte Carlo simulations on SAMPL4 molecules, and reported a mean unsigned average error of $3.0 \mathrm{kcal} / \mathrm{mol}$ in their computed MM hydration free energies using the general AMBER force field (GAFF), and a smaller error of 1.8 $\mathrm{kcal} / \mathrm{mol}$ with QM/MM corrections at the B3LYP/6-31G* level of theory. Meanwhile, König et $\mathrm{al}^{13}$ reported a RMSE of $2.3 \mathrm{kcal} / \mathrm{mol}$ in their MM-TIP3P solvation energies using the CHARMM generalized force field (CGenFF), which was reduced to $1.6 \mathrm{kcal} / \mathrm{mol}$ after $\mathrm{QM} / \mathrm{MM}$ corrections based on the non-Boltzman Bennett (NBB) method (QM/MM-NBB, which employs data from two end states to minimize the variance of the estimate of free energy differences) ${ }^{10,14}$ and the B3LYP/6-31G* level of theory.

So far, the accuracy associated with QM/MM corrections are only made possible with a high computational cost. For example, the aforementioned QM/MM NBB correction utilized only one frame for each 1ps (1000 time steps) of two MM simulation trajectories. But, with an average of 10 to 15 self-consistent field (SCF) cycles required to fully converge QM energies for each of the thousands of frames, the $Q M / M M N B B$ correction can take several times longer than the MM simulation. It is clearly desirable to develop ways to speed up the QM/MM correction part of the calculation.

We note that the QM/MM-NBB corrected hydration free energy for each SAMPL4 molecule has a standard deviation of larger than $0.3 \mathrm{kcal} / \mathrm{mol}$ (with the largest standard deviations larger than $1 \mathrm{kcal} / \mathrm{mol}$ in Table 1 of Reference ${ }^{13}$ ). With such a large standard deviation, one can question the necessity of tightly converging the QM/MM energy for each frame. In the case of density functional theory (DFT) calculations like B3LYP/6-31G*, it is certainly desirable to reduce the computer time by going through fewer SCF cycles. In the best case scenario, one avoids all SCF cycles and instead estimates the QM/MM energy (hopefully with an accuracy of about 0.1 or $0.2 \mathrm{kcal} / \mathrm{mol}$ ).

Recently, several methods have indeed been developed to estimate the QM/MM energy. Hu et al. ${ }^{15}$ developed two response kernels to describe how the electrostatic-potential-derived (ESP) charges respond to external electrostatic fields and to QM nuclear motions, and used those two kernels to compute the QM/MM energy for a given configuration. Pulay and Janowski used generalized multipole moments and polarizabilities ${ }^{16,17}$ to compute the change in QM/MM energy with an external electrostatic field due to MM atoms. More recently, we developed two multiple environment single system (MESS) QM/MM schemes ${ }^{18}$, one based on Fock marix extrapolation (MESS-E) and the other based on the inverse electronic Hessian (MESS-H), for rapidly estimating QM/MM energies for a frozengeometry QM system embedded in a dynamical MM environment. 
In this work, we will apply the MESS-E scheme to the computation of hydration free energies of SAMPL4 molecules and twelve additional small solute molecules, for which all solute molecules will be kept at their gas-phase geometry. The twelve molecules introduce additional chemical heterogeneity and include water, amino acid side chain analogs as well as structural mimics of sugars. They are also small enough to perform expensive QM/MM calculations, and their $\mathrm{pK}_{a}$ values assure charge neutrality at a $\mathrm{pH}$ of 7 .

In Section II, the MESS-E scheme will be briefly reviewed, and a scaling of the MESS-E step size will be introduced to avoid a systematic overestimation of QM/MM polarization energies. In the same section, other computational details like implicit solvent methods and MM free energy calculation set up will be presented. In Section III, for the purpose of comparison, results from four implicit solvation models will be presented: SM8 ${ }^{19}$, SM12MK, SM12CHELPG ${ }^{20}$, and SMD ${ }^{21}$. Results from MM hydration free energy simulations and QM/MM-NBB corrections (with and without applying the MESS-E scheme) will be shown and discussed in Section IV. Concluding remarks will be made in Section V.

\section{THEORY AND COMPUTATIONAL DETAILS}

\section{A. The MESS-E-QM/MM Scheme with Scaled Step Sizes}

The $\mathrm{QM} / \mathrm{MM}$ polarization energy, $\Delta E_{2}$, arises from the relaxation of occupied orbitals within the MM embedding potential that lowers the energy of (and thus stabilizes) the QM region. Following our previous work ${ }^{18}$, for a fixed-geometry QM region embedded in an MM electrostatic potential $\Delta v(\mathbf{r})$, it is defined as

$$
\Delta E_{2}=E-E_{0}-\Delta E_{1}=E-E_{0}-\left[\sum_{A \in Q M} Z_{A} \Delta v(\mathbf{A})-\int d \mathbf{r} \rho_{0}(\mathbf{r}) \Delta v(\mathbf{r})\right],
$$

where $E$ is the total energy (excluding QM/MM vdW interactions and pure MM interactions), $E_{0}$ is the gas-phase Kohn-Sham DFT energy and $\rho_{0}(\mathbf{r})$ is the corresponding electron density. $\Delta E_{1}$ is the $\mathrm{QM} / \mathrm{MM}$ permanent electrostatic interaction energy, which contains contributions from both QM nuclei (with charges of $Z_{A}$ ) and the gas-phase electron density $\rho_{0}(\mathbf{r})$. In this work, the MM electrostatic embedding potential, $\Delta v(\mathbf{r})$, is computed as a sum of contributions from MM (TIP3P) point charges $\left(\left\{q_{B}\right\}\right.$ at $\left.\mathbf{B}\right)$,

$$
\Delta v(\mathbf{r})=\sum_{B \in M M} \frac{q_{B}}{|\mathbf{r}-\mathbf{B}|}
$$

In our previous work ${ }^{18}$, we reported two schemes for estimating the QM/MM polarization energy in Eq. 1: (a) MESS-H, which is based on the electronic Hessian (also known as response kernel or stability matrix or simply energy second derivatives with respect to orbital rotations); and (b) MESS-E, which is based on Fock matrix extrapolation and 
resembles the basis set extrapolation ${ }^{22-25}$, functional jump ${ }^{26}$ and other extrapolation methods in quantum chemistry. The SAMPL4 molecules in this study contain 22 to 44 atoms, which makes the computation of gas-phase electronic Hessian (used in the MESS-H scheme) rather expensive in terms of both CPU time and memory requirement. It is possible to speed up the computation of the electron Hessian through representing it in a subspace of non-orthonormal base vectors and through invoking the resolution of the identity of approximation, which will be explored in the near future. Meanwhile, in this study, only the MESS-E scheme will be employed, and the QM/MM polarization energy (Eq. 1) will be estimated via a three-stage process:

- Gas-phase calculation. Compute gas-phase density matrix, $\mathbf{P}_{0}$, electron density, $\rho_{0}(\mathbf{r})$, and Fock matrix, $\mathbf{F}_{0}$.

- $\quad$ Training period. For the first $10 \mathrm{MM}$ configurations, which are used as a training set, fully converge Kohn-Sham DFT. Find a step size scaling factor, $\lambda$, as defined below in Eq. 3 to minimize the root-mean-square deviation of the estimated QM/MM polarization energies (in Eq. 4) from the fully converged values.

- Production period. For each MM configuration,

- $\quad$ Compute the MM electrostatic embedding potential, $\Delta v(\mathbf{r})$, and its atomic-orbital representation, $\Delta \mathbf{h}$.

- $\quad$ Form a perturbed Fock matrix,

$$
\mathbf{F}_{1}=\mathbf{F}_{0}+\lambda \Delta \mathbf{h}
$$

- $\quad$ Diagonalize the perturbed Fock matrix to obtain a perturbed density matrix $\mathbf{P}_{1}$.

- Compute the MESS-E estimation to the QM/MM polarization energy:

$$
\Delta E_{2}(\text { MESS- E- QM } / \mathrm{MM})=\mathbf{F}_{1} \cdot\left(\mathbf{P}_{1}-\mathbf{P}_{0}\right) .
$$

Within this procedure, a parameter $\lambda$ is employed to scale down the Roothaan step size in order to avoid a systematic overestimation of the QM/MM polarization energy. Specifically, the first $10 \mathrm{MM}$ configurations are used as a training set to optimize the $\lambda$ value at a given level of QM theory (i.e. density functional and basis set).

\section{B. Classical Simulations of Hydration Free Energies}

The CHARMM program ${ }^{27}$ was employed to compute the hydration free energies, using $\mathrm{CGenFF}^{28,29}$ parameters for the fixed-geometry solute molecule and TIP3P for solvent water molecules ${ }^{30}$. In all simulations, the unit cell is a truncated octahedron, containing 1492 water molecules for simulations on the primary set of SAMPL4 molecules, which are shown in Fig. 1(a). We also simulated the hydration of twelve additional small molecules: water, methanol, ethanol, methanethiol, acetamide, tetrahydrofuran, benzene, aniline, 
phenol, ethane, hexane, and cyclohexane, which are shown in Fig. 1(b), to test whether trends found from our SAMPL4 molecule calculations are applicable to common solute molecules. Amongst, methanol, ethanol, methanethiol and acetamide have been previously used as amino acid side chain analogs, ${ }^{1}$ while other molecules are selected from a previous dataset from Mobley et $\mathrm{al}^{31}$ to represent ring compounds and hydrophobic molecules. In the simulation of these small solute molecules, the unit cell contained 1687 TIP3P water molecules.

Following previous calculations ${ }^{13}$, we use the PERT module in CHARMM to gradually turn off all non-bonded interactions (electrostatic or Lennard-Jones) between the solute and solvent molecules, and the non-bonded interactions internal to the solute molecules. This alchemical mutation was done through $24 \lambda$ points: first gradually weakening electrostatic interactions $13 \lambda$ points $\left(\lambda_{\text {elec }}=1.00,0.90, \cdots, 0.20,0.10,0.05,0.00\right)$, and then gradually weakening vdW interactions $\left(\lambda_{L J}=1.00,0.95,0.90, \cdots, 0.20,0.10,0.05,0.00\right)$ using soft core.

To enhance the sampling efficiency, $\lambda$-Hamiltonian replica exchange ${ }^{32}$ was employed to conditionally swap structures between the neighboring $\lambda$ points every 1,000 time steps ( 1 ps). The solution-phase free energy difference is $\Delta G_{\text {solution }}=\sum_{i=0}^{23} \Delta G_{i, i+1}$, and the gasphase energy difference is $\Delta G_{\text {gas }}=E_{\text {gas }}\left(\lambda_{\text {elec }}=0.00 ; \lambda_{L J}=0.00\right)-E_{\text {gas }}\left(\lambda_{\text {elec }}=1.00 ; \lambda_{L J}=\right.$ 1.00). Through the thermodynamic cycle, the hydration free energy is $\Delta G_{g a s}-\Delta G_{\text {solution. }}$.

It should be noted that both the gradual switching-off of nonbonded interactions within the solute molecule and the Hamiltonian replica exchange were useful in the previous work ${ }^{13}$ for enhancing the conformational sampling for the solute molecules. In our work, neither should be absolutely necessary because the solute molecule adopts a fixed geometry. We retained the same procedure so that a direct comparison can be made to previous results. ${ }^{13}$

Each of the $24 \lambda$ points refers to a 1 ns Langevin NVT trajectory at a temperature of $300 \mathrm{~K}$. The first 100ps is the equilibration period, the remaining 900ps is divided into five 180ps segments for the computation of five hydration free energies (including $\Delta E_{g a s}$ ): $\Delta G_{m}, m=1$,

$M$ where $\mathrm{M}$ is 5 . The average hydration free energy is then $\Delta G_{\text {average }}=\frac{1}{M} \sum_{m=1}^{M} \Delta G_{m}$, and the standard deviation is $\sqrt{\sum_{m=1}^{M}\left(\Delta G_{m}-\Delta G_{\text {average }}\right)^{2} /(M-1)}$.

\section{Non-Boltzmann Bennett Procedure for Computing QM/MM Energy Corrections}

To compute the QM/MM energy correction to the MM hydration free energy, frames from the last $900 \mathrm{ps}$ of the first two MM trajectories, 0: $\left(\lambda_{\text {elec }}=1.00, \lambda_{L J}=1.00\right)$ and 1: $\left(\lambda_{\text {elec }}=\right.$ $0.90, \lambda_{L J}=1.00$ ), are collected at intervals of 100 timesteps, yielding 9,000 frames for each trajectory. Then the $\mathrm{QM} / \mathrm{MM}$ energy (without periodic boundary conditions), $U Q M / M M$, is evaluated for each frame, and the potential energy difference, $V^{b}=U^{M M}-U^{Q M / M M}$, is employed as the biasing potential. The QM/MM free energy difference between the two MM trajectories (for each of the 180ps segments) is computed via non-Boltzmann-Bennett as in Reference ${ }^{10}$ 


$$
\Delta A^{0 \rightarrow 1}=\beta^{-1} \ln \left(\frac{\left\langle f\left(\Delta U_{b w}+C\right) \exp \left(\beta V_{1}^{b}\right)\right\rangle_{1, M M}\left\langle\exp \left(\beta V_{0}^{b}\right)\right\rangle_{0, M M}}{\left\langle f\left(\Delta U_{f w}-C\right) \exp \left(\beta V_{0}^{b}\right)\right\rangle_{0, M M}\left\langle\exp \left(\beta V_{1}^{b}\right)\right\rangle_{1, M M}}\right)+C
$$

where $<>$ denotes ensemble average, $\beta$ is $1 / \mathrm{kT}, f(x)$ is the Fermi function $(1+\exp (\beta x))^{-1}$, $U_{b W}$ is the backward perturbation $U_{0}-U_{1}$, and $U_{f W}$ is the forward perturbation $U_{1}-U_{0}$. The $C$ value is solved for iteratively.

\section{Other Computational Details}

For QM/MM calculations on the MM frames, a developmental version of the Q-CHEM 4.3 software $^{33}$ was employed within the Q-CHEM/CHARMM software interface ${ }^{34}$. The gasphase geometries of all solute molecules were optimized using the $\omega \mathrm{B} 97 \mathrm{X}-\mathrm{D}$ density functional ${ }^{35}$ and the $6-311++\mathrm{G}^{* *}$ basis set. In all subsequent solvation free energy calculations, the solute molecules are fixed to these gas-phase geometries.

Several density functionals, BLYP ${ }^{36,37}, \mathrm{~B}^{2} \mathrm{LYP}^{36-38}, \mathrm{PBE} 0^{39}, \mathrm{M} 06-2 \mathrm{X}^{40}$, and $\omega \mathrm{B} 97 \mathrm{X}-\mathrm{D}^{35}$, were applied to all solute molecules using the $6-31 \mathrm{G}^{*}$ basis set. Numerical integration was performed with an atom-centered grid ${ }^{41}$ with 99 radial points and 590 angular points. For the twelve small solute molecules, two additional QM methods were applied: the Coulombattenuated MP2 method ${ }^{42,43}$ using the aux-cc-pVDZ basis and rimp2-aug-cc-pVDZ auxilary basis set (i.e. ATT-RIMP2/aug-cc-pVDZ); and the XYGJ-OS functional ${ }^{44,45}$ within the 6-311+G(3df,2p) basis set.

For comparison, four Minnesota implicit solvent models were also applied to all solute molecules: SM8 ${ }^{19}$, SM12MK, SM12CHELPG ${ }^{20}$, and SMD ${ }^{21}$, all with the $6-31 G^{*}$ basis set. Three out of the four models, SM8, SM12MK, SM12CHELPG, employ the generalized Born models to describe the electrostatic component of solvation free energy with different sets of charges: CM4 charges for SM8 ${ }^{46}$; Merz-Kollman ESP charges ${ }^{47,48}$ for SM12MK; CHELPG charges ${ }^{49}$ (as implemented in Q-CHEM ${ }^{50,51}$ ) for SM12CHELPG. SMD is a polarized continuum model, where the electrostatic contribution to the solvation free energies are computed via a set of screening charges on the molecular van der Waals surface. In all four models, cavity-dispersion-solvent-structure (CDS) terms are also added to describe other contributions to the solvation free energy.

In all cases, the mean signed error (MSE) is computed as

$$
M S E=\frac{1}{K} \sum_{k=1}^{K}\left(\Delta G_{k}(\text { computed })-\Delta G_{k}(\operatorname{expt})\right)
$$

where $k$ runs over the solute molecules. The root mean square error (RMSE) is computed 


$$
R M S E=\sqrt{\frac{1}{K} \sum_{k=1}^{K}\left(\Delta G_{k}(\text { computed })-\Delta G_{k}(\exp t)\right)^{2}} .
$$

Note that out of the SAMPL4 molecules, the experimental hydration free energy for molecule $\mathbf{1}$ is actually an extrapolation from a sub-cooled liquid based on non-experimental values. ${ }^{52}$ Furthermore, molecules $\mathbf{2 2}, \mathbf{2 3}$, and $\mathbf{2 4}$ contain protonizable groups, which makes their hydration free energies strongly dependent on their $\mathrm{pK}_{a}$ 's. Unfortunately there are no experimental $\mathrm{pK}_{a}$ 's for those molecules. Since we lack the required expertise to evaluate the usability of the experimental data for those molecules, we provide the reader with two sets of MSE and RMSE values, where MSE and RMSE cover the entire set of molecules, and MSE' and RMSE' exclude the controversial molecules 1, 22, 23 and 24. The $\mathrm{R}^{2}$ values cover the entire set of 21 molecules.

\section{RESULTS FROM IMPLICIT SOLVENT CALCULATIONS}

\section{A. SAMPL4 molecules}

SM8, SM12MK, SM12CHELPG, and SMD hydration free energies with five density functionals (BLYP, B3LYP, PBE0, M06-2X, and $\omega$ B97X-D) and the 6-31G* basis set for SAMPL4 molecules at their gas-phase $\omega \mathrm{B} 97 \mathrm{X}-\mathrm{D} / 6-311++\mathrm{G}^{* *}$ geometries are listed in Tables S1-S4, and the average errors are collected in Table I. By computing the hydration free energies at the gas-phase geometry, we have neglected the effects from geometry relaxations in the condensed phase and the corresponding vibrational zero-point energy differences.

For SM8, the MSE errors are shown in Table I to be all positive, meaning that the solvation free energies are all underestimated. The smallest error occurs with the $\omega \mathrm{B} 97 \mathrm{X}-\mathrm{D}$ functional (MSE' $=0.42 \mathrm{kcal} / \mathrm{mol}$; RMSE' $=0.72 \mathrm{kcal} / \mathrm{mol}$ ) and the PBE0 functional $($ MSE' $=0.43 \mathrm{kcal} /$ $\mathrm{mol}$; RMSE' $=0.75 \mathrm{kcal} / \mathrm{mol}$ ). The B3LYP results have slightly larger error (MSE' $=0.76$ $\mathrm{kcal} / \mathrm{mol}$; RMSE' $=0.96 \mathrm{kcal} / \mathrm{mol})$. BLYP errors $\left(\mathrm{MSE}^{\prime}=0.84 \mathrm{kcal} / \mathrm{mol} ; \mathrm{RMSE}{ }^{\prime}=1.04 \mathrm{kcal} /\right.$ $\mathrm{mol})$ and M06-2X (MSE'= $1.03 \mathrm{kcal} / \mathrm{mol}$; RMSE' $=1.21 \mathrm{kcal} / \mathrm{mol})$ are even larger, but these errors are overall still reasonable. MSE and RMSE values, which also include molecules 1, 22, 23 and 24, lead to the same overall picture that PBE0 and $\omega \mathrm{B} 97 \mathrm{X}$-D yield the most accurate hydration free energies. The $\mathrm{R}^{2}$ values are 0.95 and 0.96 , suggesting an overall good correlation between SM8 hydration energies and experimental values.

With the other three Minnesota solvation models, SM12MK, SM12CHELPG and SMD, the $\mathrm{R}^{2}$ values remain around 0.92 and 0.96 . One can note that the MSE and MSE' values become systematically more negative for functionals containing larger ratios of HartreeFock exchange. With SM12MK, for example, the MSE' errors are: $0.46 \mathrm{kcal} / \mathrm{mol}$ (BLYP); $-0.19 \mathrm{kcal} / \mathrm{mol}$ (B3LYP); $-0.75 \mathrm{kcal} / \mathrm{mol}$ (PBE0); $-0.71 \mathrm{kcal} / \mathrm{mol}$ (M06-2X); and -0.85 $\mathrm{kcal} / \mathrm{mol}(\omega \mathrm{B} 97 \mathrm{X}-\mathrm{D})$.

This systematic trend reflects that larger ratios of Hartree-Fock exchange usually leads to more charge separation in a molecule, and thus leads to larger electrostatic potentials (in 
terms of the absolute values) around the molecule. Both SM12MK and SM12CHELPG models are based on ESP-fitted charges (MK and CHELPG), thus yielding more negative electrostatic energies within the generalized Born model. With the SMD model, the surface charges are employed to cancel the solute electrostatic potential, and, with higher ratio of Hatree-Fock exchange, they can also lead to overall more negative electrostatic energies.

In terms of RMSE and RMSE' values with the SM12MK model, BLYP (RMSE $=1.19 \mathrm{kcal} /$ mol; RMSE' $=0.99 \mathrm{kcal} / \mathrm{mol})$ and B3LYP $($ RMSE $=1.02 \mathrm{kcal} / \mathrm{mol} ;$ RMSE' $=1.07 \mathrm{kcal} /$ mol) are more accurate than three other functionals. With SM12CHELPG, B3LYP outperforms all four other functionals with a RMSE of $0.91 \mathrm{kcal} / \mathrm{mol}$ and RMSE' of 0.82 $\mathrm{kcal} / \mathrm{mol}$. With the SMD model, on the other hand, PBE0, M06-2X and $\omega$ B97X-D yielded smaller RMSE and RMSE' values of between 1.01 and $1.22 \mathrm{kcal} / \mathrm{mol}$. So, out of these combinations, the best performance comes from the SM12CHELPG model using the B3LYP functional.

\section{B. Twelve small molecules}

The hydration free energies of these molecules based on implicit solvent model are collected in Tables S5-S8, which all correlate reasonably well with experiments with $\mathrm{R}^{2}$ values of 0.91-0.97. As shown in Table II, the SM8 results with all five functionals are roughly equally accurate, with a RMSE value of between 1.06 and $1.11 \mathrm{kcal} / \mathrm{mol}$.

With the SM12MK, SM12CHELPG, and SMD models, a systematic change (with the functionals) in the hydration free energy is again observed. With SM12MK, for example, the MSE errors are: $-0.35 \mathrm{kcal} / \mathrm{mol}$ (BLYP); $-0.72 \mathrm{kcal} / \mathrm{mol}$ (B3LYP); $-1.08 \mathrm{kcal} / \mathrm{mol}$ (PBE0); $-1.09 \mathrm{kcal} / \mathrm{mol}(\mathrm{M} 06-2 \mathrm{X})$; and $-1.13 \mathrm{kcal} / \mathrm{mol}(\omega \mathrm{B} 97 \mathrm{X}-\mathrm{D})$.

Like the SAMPL4 molecules, this systematic change in the hydration free energies for small molecules is caused by the change in the electronic structure with the density functionals. As shown in Table S9, seven out of the twelve molecules (water, methanol, ethanol, methanethiol, acetamide, tetrahydrofuran, and phenol) acquire larger gas-phase dipole moments from BLYP to B3LYP, PBE0, M06-2X, and $\omega$ B97X-D functionals, and thus exhibit larger electrostatic potentials (in terms of the absolute values) outside the molecule, yielding a more negative electrostatic component for the hydration energy. Molecular dipole moments are zero or quite small for four other molecules (benzene, ethane, hexane, and cyclohexane). For those four molecules (and aniline), when the ratio of Hartree-Fock exchange interaction increases in the density functional, higher-order multipole moments are expected to enhance the electrostatic potential outside the molecule.

In terms of RMSE errors with the SM12MK model, BLYP $(0.79 \mathrm{kcal} / \mathrm{mol})$ and B3LYP $(1.02$ $\mathrm{kcal} / \mathrm{mol})$ are again the most accurate. With the SM12CHELPG model, BLYP $(0.75 \mathrm{kcal} /$ $\mathrm{mol})$ and B3LYP $(0.94 \mathrm{kcal} / \mathrm{mol})$ also outperform the three other functionals. This is again reversed with the SMD model: with an RMSE error of 0.77 or $0.78 \mathrm{kcal} / \mathrm{mol}$, PBE0, M06-2X and $\omega$ B97X-D perform better than BLYP and B3LYP. 


\section{Choice of implicit solvation models and functionals}

Overall, for both sets of molecules, SM12CHELPG performs better than SM12MK. But the relative performance among SM8, SM12CHELPG, and SMD is highly dependent upon the functional. For these two sets of molecules, it seems that SM12CHELPG is best used with BLYP and B3LYP functionals, while SMD is better used with PBE0, M06-2X and $\omega$ B97XD. However, this should not be over-generalized, because the two test sets are rather small and because the implementation of SM12MK, SM12CHELPG, and SMD models can vary significantly from package to package, especially when it comes to surface construction and discretization.

Meanwhile, it would be desirable to reduce the functional dependence of the SM12MK, SM12CHELPG, and SMD solvation free energies. Since stronger electrostatic interactions might arise with higher ratios of Hartree-Fock exchange, one can imagine at least two possible solutions: (1) making the vdW radii in the SM12MK and SMD models density dependent, where one can potentially follow the strategy of scaling vdW radii in the manybody dispersion (MBD) method of Tkatchenko et al. ${ }^{53,54}$ or (2) making the dispersion term of the CDS energy contributions density dependent.

\section{RESULTS FROM EXPLICIT SOLVENT CALCULATIONS}

\section{A. $\lambda$ Values}

As mentioned in Section IIB, a scaling factor $(\lambda)$ is employed in MESS-E-QM/MM calculations to avoid a systematic overestimation of $\mathrm{QM} / \mathrm{MM}$ polarization energies. In our calculations, the DFT/MM calculations were fully converged for the first $10 \mathrm{MM}$ frames of each trajectory. The $\lambda$ value is then optimized for MESS-ES-QM/MM calculations to best reproduce these converged DFT/MM energies.

The optimal $\lambda$ values from all test cases are plotted against the HOMO-LUMO gaps in Fig. 2 , with the actual $\lambda$ values and HOMO-LUMO gaps listed in Table S10-S13. It is clear from Fig. 2 that smaller HOMO-LUMO gaps tend to lead to smaller $\lambda$ values. In other words, smaller MESS-E step sizes are taken with BLYP than other functionals, while the step sizes are the largest with M06-2X and $\omega$ B97X-D. For example, as shown in Table S10, HOMOLUMO gaps of molecule 1 of the SAMPL4 blind test are: 0.226 (BLYP); 0.310 (B3LYP); 0.336 (PBE0); 0.430 (M06-2X); and 0.481 ( $\omega$ B97X-D). Meanwhile, the $\lambda$ values in Table S12 for this molecule are: 0.830 (BLYP); 0.890 (B3LYP); 0.900 (PBE0); 0.960 (M06-2X); and $0.980(\omega \mathrm{B} 97 \mathrm{X}-\mathrm{D})$.

\section{B. MESS-QM/MM-NBB versus Full QM-MM-NBB}

QM/MM-NBB-corrected solvation energies are collected for the blind SAMPL4 data set in Table III, using both fully-converged M06-2X/TIP3P energies and MESS-E-QM/MM energies. The MESS-E-QM/MM-NBB solvation energies deviate from fully converged QM/MM-NBB values by a MSE' of $0.09 \mathrm{kcal} / \mathrm{mol}$ and a RMSE' of $0.18 \mathrm{kcal} / \mathrm{mol}$. This deviation is comparable to the standard deviations for the computed solvation free energies, which is usually around 0.2 or $0.3 \mathrm{kcal} / \mathrm{mol}$. 
Among molecules 2 through 21, the smallest deviations occur with molecule 16 (5.04 vs $5.07 \mathrm{kcal} / \mathrm{mol})$ and molecule $3(7.52$ vs $7.47 \mathrm{kcal} / \mathrm{mol})$. And the largest deviations occur with molecule 9 (10.57 vs $10.27 \mathrm{kcal} / \mathrm{mol})$ and molecule 6 (10.87 vs $10.37 \mathrm{kcal} / \mathrm{mol})$.

For the twelve small molecules, two sets of full QM/MM energy evaluations were performed, BLYP/TIP3P and M06-2X/TIP3P, and the results are shown in Table IV. With BLYP, the MESS-E-QM/MM-NBB corrected solvation energies differ from the fully converged QM/MM-NBB corrected values by a MSE of $0.05 \mathrm{kcal} / \mathrm{mol}$ and a RMSE of 0.15 $\mathrm{kcal} / \mathrm{mol}$. The smallest deviation occurs with tetrahydrofuran (both $3.29 \mathrm{kcal} / \mathrm{mol}$ ) and ethane (both $2.04 \mathrm{kcal} / \mathrm{mol})$, while the largest deviation occurs with acetamide $(-11.68 \mathrm{vs}$ $-11.19 \mathrm{kcal} / \mathrm{mol}$ ). With M06-2X, the MESS-E-QM/MM-NBB corrected solvation energies differ from the fully converged QM/MM-NBB corrected values by a MSE of $0.06 \mathrm{kcal} / \mathrm{mol}$ and a RMSE of $0.10 \mathrm{kcal} / \mathrm{mol}$. The smallest deviation occurs with ethane (both $2.02 \mathrm{kcal} /$ $\mathrm{mol})$, while the largest deviation occurs again with acetamide $(-13.62 \mathrm{vs}-13.37 \mathrm{kcal} / \mathrm{mol})$.

\section{The significance of $\mathrm{QM} / \mathrm{MM}$ polarization energy contribution to the hydration free energy}

If we keep only QM/MM permanent electrostatics, $\Delta E_{1}$ in Eq. 1, and neglect QM/MM polarization energy, $\Delta E_{2}$, the $\mathrm{QM} / \mathrm{MM}-\mathrm{NBB}$ correction to the hydration free energies change rather significantly. As shown for the twelve molecules in Table IV, the computed QM/MMNBB-corrected hydration free energy will differ by a MSE of $1.81 \mathrm{kcal} / \mathrm{mol}$ with BLYP functional applied to the QM region, and a MSE of $2.01 \mathrm{kcal} / \mathrm{mol}$ with M06-2X functional. The RMSE values are also large: $2.18 \mathrm{kcal} / \mathrm{mol}$ with BLYP and $2.41 \mathrm{kcal} / \mathrm{mol}$ with M06-2X.

This means that QM/MM polarization energies cannot be neglected in the QM/MM-NBB hydration free energy calculations. On the other hand, if one adopts a condensed-phase reference (with an implicit model or an average solvent potential) instead of a gas-phase one, the MSE values might get reduced. But we expect the deviations there to be still significant.

\section{Results for the Blind SAMPL4 Hydration Free Energy Test Set}

1. Computational Time-The computational time for hydration free energy calculations on the SAMPL4 molecules are listed in Table V. Since 1492 water molecules are included in the calculations, it takes roughly the same amount of computer time | about 2,500 CPU hours using a single core of an Intel Xeon E5520 2.27GHz processor | to compute the MM solvation free energies for each solute. Most of the cost arises from the generation of the 24 parallel MM trajectories (each $1 \mathrm{~ns}$ long), with a small overhead due to the computation of $\mathrm{MM}$ energies for frames on neighboring trajectories for $\lambda$-Hamiltonian replica exchange.

Even for the smallest SAMPL4 solute (molecule 5), it takes about 10,000 CPU hours to compute the QM/MM energies for all 20,000 frames, and this cost increases to about 65,000 CPU hours for the largest SAMPL4 solute (molecule 24). Overall, the QM/MM corrections can be 4 to 26 times more expensive than the MM stage of the calculation.

With the current implemention of MESS-E-QM/MM, the Q-CHEM program is called for each frame to evaluate the current MM embedding potential, and to then compute the permanent electrostatic energy and the MESS-E estimation for the QM/MM polarization energy. For 18,000 frames, this only takes about 160 to $300 \mathrm{CPU}$ hours, which is 60 to 200 
faster than the full QM/MM calculations. Compared to the MM stage of the calculation, the MESS-E-QM/MM correction only adds $7 \%$ to $12 \%$ of additional cost. Clearly, MESSE$\mathrm{QM} / \mathrm{MM}$ provides a very fast way to compute the QM/MM-NBB correction to MM hydration free energies for rigid solute molecules.

2. Accuracy of Hydration Free Energies-In Table VI, we list the experimental hydration free energies for the blind SAMPL4 set, the fixed-geometry-solute MM hydration free energies (F-MM-TIP3P), and the corresponding MESS-E-QM/MM-NBB corrected hydration free energies using the Non-Boltzmann-Bennett algorithm and five different density functionals (BLYP, B3LYP, PBE0, M06-2X, $\omega$ B97X-D).

The MM hydration free energies with fixed-geometry solute molcules yielded a MSE' of $-1.14 \mathrm{kcal} / \mathrm{mol}$ and a RMSE' of $1.79 \mathrm{kcal} / \mathrm{mol}$. This is comparable to the MSE' value of $-0.83 \mathrm{kcal} / \mathrm{mol}$ and a RMSE' of $1.75 \mathrm{kcal} / \mathrm{mol}$ (also averaged over molecules 2 through $\mathbf{2 1}$ ) obtained from flexible solute geometry simulations ${ }^{13}$. The RMSE (including molecules $\mathbf{1}$, $\mathbf{2 2}, \mathbf{2 3}$, and 24 ) is $2.33 \mathrm{kcal} / \mathrm{mol}$, which is also close to the reported RMSE of $2.3 \mathrm{kcal} / \mathrm{mol}$ using completely flexible solutes. ${ }^{13}$ This indicates that the SAMPL4 blind data set is not very sensitive to conformational entropy.

In Table VI, one can also observe a systematic change: with few exceptions, the MESSEQM/MM corrected hydration free energies become more negative with higher ratios of Hatree-Fock exchange. The MSE' values are: $-0.55 \mathrm{kcal} / \mathrm{mol}$ (BLYP); $-1.35 \mathrm{kcal} / \mathrm{mol}$ (B3LYP); $-1.87 \mathrm{kcal} / \mathrm{mol}$ (PBE0); $-1.90 \mathrm{kcal} / \mathrm{mol}(\mathrm{M} 06-2 \mathrm{X})$ and $-2.06 \mathrm{kcal} / \mathrm{mol}(\omega \mathrm{B} 97 \mathrm{X}-$ D). The corresponding RMSE' values are: $1.02 \mathrm{kcal} / \mathrm{mol}$ (BLYP); $1.63 \mathrm{kcal} / \mathrm{mol}$ (B3LYP); $2.10 \mathrm{kcal} / \mathrm{mol}$ (PBE0); $2.15 \mathrm{kcal} / \mathrm{mol}(\mathrm{M} 06-2 \mathrm{X}) ; 2.29 \mathrm{kcal} / \mathrm{mol}(\omega \mathrm{B} 97 \mathrm{X}-\mathrm{D})$. For the entire set of 21 molecules, BLYP also yielded a rather small MSE value of $0.10 \mathrm{kcal} / \mathrm{mol}$ and the smallest RMSE value of $1.95 \mathrm{kcal} / \mathrm{mol}$. So out of five functionals, BLYP clearly leads to the most accurate MESS-QM/MM-NBB corrected hydration free energies, which indicates that BLYP might be the most compatible functional with the TIP3P solvent model. This was also observed by Shaw et al ${ }^{55}$, who computed the free energy for the perturbation of a QM water molecule into a MM water molecule in bulk MM solvent. The accuracy of BLYP/TIP3P corrected hydration free energies is also comparable to the accuracy of implicit solvent models presented above in Section III.

As was mentioned in Eq. 1, when a fixed-geometry solute molecule is moved from one frame to another, its energy changes contain two contributions: QM/MM permanent electrostatics $\left(\Delta E_{1}\right)$ and $\mathrm{QM} / \mathrm{MM}$ polarization $\left(\Delta E_{2}\right)$. Further we found that, when different functionals are employed, the main change would come from the permanent electrostatics contribution. Here, similar to the observation for the implicit solvent models, higher ratios of Hartree-Fock exchange cause larger charge separation in the gas-phase solute electronic charge, therefore enhancing the electrostatic potential (from the gas-phase electron structure) at external MM sites, thus causing the permanent $\mathrm{QM} / \mathrm{MM}$ electrostatic interaction to be more significant.

Overall, it is clear from Table VI that QM/MM-NBB corrections can improve MM results. MSE, RMSE, MSE' and RMSE' are all improved upon the MESS-E-QM/MM-NBB 
correction using BLYP/TIP3P. For example, the RMSE' value is reduced from $1.79 \mathrm{kcal} / \mathrm{mol}$ to $1.02 \mathrm{kcal} / \mathrm{mol}$. The $\mathrm{R}^{2}$ value is increased from 0.72 to $0.82-0.87$ upon $\mathrm{QM} / \mathrm{MM}$ correction.

\section{E. Results for Twelve Small Solute Molecules}

Experimental hydration free energies for the twelve small solute molecules are collected from several references ${ }^{1-3,31,56,57}$ and listed in Table VII. Also shown in the table are the hydration free energies from fixed-solute-geometry MM simulations, which have a MSE of $0.77 \mathrm{kcal} / \mathrm{mol}$ and a RMSE of $0.97 \mathrm{kcal} / \mathrm{mol}$ and therefore are of good quality.

When the MESS-E-QM/MM-NBB correction is added, the MSE values are: $-0.18 \mathrm{kcal} / \mathrm{mol}$ (BLYP); $-0.66 \mathrm{kcal} / \mathrm{mol}$ (B3LYP); $-1.05 \mathrm{kcal} / \mathrm{mol}$ (PBE0); $-1.12 \mathrm{kcal} / \mathrm{mol}$ (M06-2X); and $-1.15 \mathrm{kcal} / \mathrm{mol}(\omega \mathrm{B} 97 \mathrm{X}-\mathrm{D})$. Again, one sees more negative hydration free energies with higher ratios of Hartree-Fock exchange. For these twelve molecules, as shown in Table S14, the energy change between frames is clearly dominated by QM/MM permanent electrostatics $\left(\Delta E_{1}\right)$. Take the water molecule for instance. Compared to the value using the BLYP functional, its $\Delta E_{1}$ gets reduced by an average of $-0.65 \mathrm{kcal} / \mathrm{mol}$ with B3LYP, -1.01 $\mathrm{kcal} / \mathrm{mol}$ with PBE0, and $-1.26 \mathrm{kcal} / \mathrm{mol}$ with M06-2X and $\omega$ B97X-D. Meanwhile, the changes in the QM/MM polarization energy $\left(\Delta E_{2}\right)$ is about ten times smaller: $0.07 \mathrm{kcal} / \mathrm{mol}$ with B3LYP; $0.10 \mathrm{kcal} / \mathrm{mol}$ with PBE0; $0.11 \mathrm{kcal} / \mathrm{mol}$ with M06-2X; and $0.17 \mathrm{kcal} / \mathrm{mol}$ with $\omega \mathrm{B} 97 \mathrm{X}-\mathrm{D}$. There, the $\mathrm{QM} / \mathrm{MM}$ polarization energy, which is always negative (between 0 and $-12 \mathrm{kcal} / \mathrm{mol}$ for these twelve molecules), becomes slightly less negative with increasing ratios of Hartree-Fock exchange.

As shown in Table VII, the RMSE in hydration free energies for these solute molecules are: $1.00 \mathrm{kcal} / \mathrm{mol}$ with BLYP; $1.25 \mathrm{kcal} / \mathrm{mol}$ with B3LYP; $1.59 \mathrm{kcal} / \mathrm{mol}$ with PBE0; 1.64 $\mathrm{kcal} / \mathrm{mol}$ with M06-2X; and $1.69 \mathrm{kcal} / \mathrm{mol}$ with $\omega \mathrm{B} 97 \mathrm{X}-\mathrm{D}$. Thus, BLYP, the least sophisticated functional out of the five, again produces the most accurate hydration free energies. Interestingly, BLYP is slightly worse than the MM-results in terms of the RMSE values (1.00 vs $0.96 \mathrm{kcal} / \mathrm{mol})$; but it has a more favorable MSE (-0.18 vs $0.77 \mathrm{kcal} / \mathrm{mol})$.

Since these twelve small molecules are relatively small, two advanced $a b$ initio quantum chemistry methods can also be employed: ATT-RIMP2/aug-cc-pVDZ, which is a Coulombattenuated MP2 method; ${ }^{42,43}$ and XYGJ-OS/6-311+G(3df,2p), which is a double-hybrid density functional. ${ }^{44,45}$ As shown in Table VIII, the ATT-RIMP2/aug-cc-pVDZ method yields a MSE of $-1.20 \mathrm{kcal} / \mathrm{mol}$ and a RMSE of $1.80 \mathrm{kcal} / \mathrm{mol}$. Meanwhile, the XYGJ-OS method yields a MSE of $-1.46 \mathrm{kcal} / \mathrm{mol}$ and a RMSE of $2.06 \mathrm{kcal} / \mathrm{mol}$. So these two methods produce even less accurate hydration free energies than the previous five density functionals. This indicates that, when the solute molecule retains a fixed geometry, more sophisticated $a b$ initio quantum chemistry methods alone do not necessarily lead to more accurate hydration free energies.

In QM/MM calculations with a fixed-geometry QM region, there exists a balance between two non-bonded interactions: permanent electrostatics and vdW. As more sophisticated $a b$ initio quantum chemistry methods are applied to the QM region, the quality of QM/MM electrostatics might be improved. But this might be counter balanced by the treatment of the 
vdW interaction. In the future, it is desirable to develop more sophisticated schemes for the QM/MM vdW interaction and make it explicitly dependent on the electron structure, which hopefully makes the final results less dependent on the choice of density functionals.

\section{F. QM/MM Sampling Frequency}

As mentioned in Section II C, frames are collected from MM trajectories at intervals of 100 time steps (or $0.1 \mathrm{ps}$ ). In order to evaluate the influence of the sampling frequency on the free energy estimate, we also sub-sampled the data according to different intervals: 200, 500, and 1000 time steps $(0.2,0.5$ and $1.0 \mathrm{ps})$. This reduces the total number of samples from 10000 to 5000, 2000 or 1000 data points for each $1 \mathrm{~ns}$ MM trajectory. The corresponding MESS-EQM/MM-NBB corrected hydrations are listed in Tables S15, S16, S17 and S18.

In Table S15, which lists BLYP/TIP3P results for SAMPL4 molecules, significantly larger standard deviations are found for molecules $2,10,11,12,13,15$, and 21 with longer sampling intervals. For example, for molecule 2, the standard deviation is $0.13 \mathrm{kcal} / \mathrm{mol}$ with 100 time step intervals, and $0.32 \mathrm{kcal} / \mathrm{mol}$ with 1000 time step intervals. This is expected because longer intervals lead to fewer datapoints for the QM/MM-NBB free energy calculation. The QM/MM-corrected hydration free energy changes by no more than 0.1 $\mathrm{kcal} / \mathrm{mol}$ for 12 out of the 21 molecules $(\mathbf{2}, 3,4,10,11,12,13,16,17,19,20$, and 21$)$. For example, the hydration free energy for molecule 2 ranges from 3.08 to $3.18 \mathrm{kcal} / \mathrm{mol}$. For five other molecules $(\mathbf{1}, \mathbf{6}, \mathbf{2 2}, \mathbf{2 3}$, and $\mathbf{2 4})$, the hydration free energies changes by no more than $0.2 \mathrm{kcal} / \mathrm{mol}$. The largest changes occur for the remaining four molecules $(\mathbf{5}, \mathbf{9}, \mathbf{1 4}$, and 15). For example, for molecule 9 , the hydration energy ranges from 8.70 to $9.38 \mathrm{kcal} / \mathrm{mol}$. This is likely due to the relatively smaller overlaps between the MM and QM/MM surfaces for these molecules. For all 21 molecules, the MSE and RMSE values change by no more than $0.09 \mathrm{kcal} / \mathrm{mol}$, and the $\mathrm{R}^{2}$ values stay constant at 0.82 or 0.83 . Very similar trends can be observed in Table S16, which collects B3LYP/TIP3P results for the SAMPL4 molecules. The hydration energies of four molecules $(\mathbf{1}, \mathbf{5}, \mathbf{9}, \mathbf{1 5})$ vary by more than $0.2 \mathrm{kcal} / \mathrm{mol}$. The overall MSE and RMSE values change by no more than $0.11 \mathrm{kcal} / \mathrm{mol}$, and $\mathrm{R}^{2}$ ranges from 0.84 to 0.87 .

For the twelve smaller solute molecules, the results in Tables S17 (with BLYP/TIP3P) and S18 (with B3LYP/TIP3P) yield a similar picture. For seven molecules (water, methanol, ethanol, benzene, ethane, hexane, and cyclohexane), the computed hydration free energies vary by no more than $0.1 \mathrm{kcal} / \mathrm{mol}$ with different sampling frequencies. For three other molecules (tetrahydrofuran, phenol, and aniline), the computed hydration free energies change by no more than $0.2 \mathrm{kcal} / \mathrm{mol}$. A larger change between 0.3 and $0.4 \mathrm{kcal} / \mathrm{mol}$ is observed only for methanethiol and acetamide. Overall, with both functionals, the MSE and RMSE values change no more than $0.13 \mathrm{kcal} / \mathrm{mol}$ with different sampling frequencies and the $\mathrm{R}^{2}$ value stays constant at 0.94 or 0.95 .

This suggests that, overall, the free energy estimate is not very sensitive to the sampling intervals within the range of 100 to 1000 time steps. Since the MSE and RMSE values change by no more than than $0.13 \mathrm{kcal} / \mathrm{mol}$, this does not change the observation that, between the two functionals, BLYP/TIP3P yields more accurate QM/MM-NBB corrected hydration free energies. 


\section{DISCUSSION}

The MESS-E-QM/MM method presented in this work keeps the solute molecules frozen at their gas-phase geometry. When BLYP/TIP3P is used, this method has been shown to yield reasonably accurate hydration free energies for not-so-flexible molecules including the SAMPL4 molecules and a dozen smaller solutes. It is also expected to work well for computing the $\mathrm{QM} / \mathrm{MM}$-corrected binding free energy of rigid ligand molecules (such as steroids) to receptors, where a continuum model for the MM environment is definitely inappropriate.

However, for cases where the QM region is flexible, the MESS-E-QM/MM method is expected to yield larger errors, because it neglects conformational entropy. There are three potential remedies:

- $\quad$ For molecules with multiple rotational isomers it is possible to perform a MESSE-QM/MM simulation for each low-energy rotational substate and then combine the data according to a scheme proposed by Straatsma and McCammon. ${ }^{58}$ Following recent work, ${ }^{11,59}$ it is also possible to account for the free energy costs of imposing constraints on the bonded terms by performing harmonic analyses and correcting for the Jacobian factors of each frame of the trajectory. The required Hessians can be approximated at the MM level due to the stiffness of the bonded terms.

- $\quad$ Relaxing the solute geometry for one step at regular intervals (e.g., every 10,000 time steps) during the MM free energy simulation. This is similar to the microiterative approach, ${ }^{60}$ sequential sampling, ${ }^{15}$, and hybrid differential relaxation algorithm. ${ }^{61}$ If the $\mathrm{QM} / \mathrm{MM}$ sampling frequency remains at 100 timesteps, then during each interval there will be 100 frames with identical solute geometry where MESS-E-QM/MM can be applied to reliably estimate QM/MM energies. Since the HOMO-LUMO gap is expected to be insensitive to molecular geometry, we expect the same scaling factor for MESS-E can be applied for all solute geometries.

- $\quad$ Combining MESS-E (which approximates the response of the solute molecule to external perturbations from solute molecules) and accurate/efficient models for mapping the solute potential energy surface (i.e., energy changes due to internal pertubations - solute geometry relaxation). There, the solute potential energy surface can be described either by a sophisticated force field ${ }^{62-64}$ or interpolation methods. ${ }^{65,66}$ With this approach, one also has to account for the coupling between responses to external perturbations and those due to internal perturbations.

These three approaches will be compared in future studies.

\section{CONCLUSIONS}

In this work, we computed hydration free energies for the blind SAMPL4 test set and twelve small molecules using a fixed gas-phase geometry. Three different levels of theory were 
applied: (a) classical molecular dynamics based free energy simulations with $24 \lambda$ points; (b) MESS-E-QM/MM-NBB corrections with explicit solvents; and (c) Minnesota implicit solvent models (SM8. SM12MK, SM12CHELPG, and SMD).

The main observations are:

- The MESS-E-QM/MM-NBB correction is computationally 60 to 200 times faster than the full QM/MM-NBB correction for the blind SAMPL4 test set. It provides results that deviate from fully-converged QM/MM-NBB results only by a RMSE of $0.18 \mathrm{kcal} / \mathrm{mol}$ for the SAMPL4 dataset, and by a smaller RMSE of 0.10 or $0.15 \mathrm{kcal} / \mathrm{mol}$ for the twelve small molecules.

- $\quad$ Compared to the MM stage of the calculation, the MESS-E-QM/MM-NBB correction only adds $7 \%$ to $12 \%$ additional cost. MESS-E-QM/MM provides a very fast way to compute the $\mathrm{QM} / \mathrm{MM}-\mathrm{NBB}$ correction to $\mathrm{MM}$ hydration free energies for rigid solute molecules.

- $\quad$ The MESS-E-QM/MM-NBB corrected hydration energies become systematically more negative with higher ratios of Hartree-Fock exact exchange, i.e. from BLYP to B3LYP to PBE0 to M06-2X and to $\omega \mathrm{B} 97 \mathrm{X}-\mathrm{D}$. This reflects stronger and stronger permanent $\mathrm{QM} / \mathrm{MM}$ electrostatic interactions with higher Hartree-Fock exchange. Out of these five functionals, BLYP is most compatible with the TIP3P solvent model and leads to the most accurate hydration free energies for molecules in this study. More sophisticated quantum chemistry methods like ATT-RIMP2 and XYGJ-OS are even less compatible with the TIP3P solvent model and lead to less accurate hydration free energies for the twelve small molecules.

- $\quad$ The $\mathrm{QM} / \mathrm{MM}$ polarization energies contribute significantly to the $\mathrm{QM} / \mathrm{MM}-\mathrm{NBB}$ correction to the MM hydration free energies. If the $\mathrm{QM} / \mathrm{MM}$ polarization energies are simply neglected, the computed hydration free energies can change by an average of $2 \mathrm{kcal} / \mathrm{mol}$ for the twelve small molecules.

- $\quad$ The implicit solvent methods in general provide results with comparable accuracy. For three models, SM12MK, SM12CHELPG, and SMD, the implicit solvation free energies also exhibited a systematic shift with increasing ratios of Hartree-Fock exchange.

At the same time, besides the frozen-geometry issue discussed in the last section, this work has other limitations:

- $\quad$ So far, MESS-E-QM/MM has only been applied to the estimation of QM/MM energies. It has to be extended to QM/MM gradient calculations, which will be necessary for driving QM/MM dynamics and for computing rigid-body ethalpic/ entropic corrections mentioned above.

- $\quad$ So far we have applied the MESS-E-QM/MM scheme on charge-neutral systems, and its application to charged systems has yet to be investigated.

and we are working to address some of these limitations. 


\section{Supplementary Material}

Refer to Web version on PubMed Central for supplementary material.

\section{Acknowledgments}

YS is supported by NIH grant GM096678-02 and DOE grant No. DE-SC0011297. GK, FCP, AS, BTM, and BRB are supported by the Intramural Research Program of the NIH, NHLBI. HLW would like to acknowledge support from the National Science Foundation under CHE-1464946. GK and YS thank Dr. Jing Huang for his help with optimizing CGenFF force field parameters for aniline. Computational resources and services used in this work were partially provided by the LoBoS cluster of the National Institutes of Health. YS thanks Drs. Dieter Cremer, Robert DiStasio, Teresa Head-Gordon, Michael Jones, Ken Jordan, Elfi Kraka, Daniel Lambrecht, Richard Pastor, Edina Rosta, Alex Sodt, Peng Tao, and Richard Venable for helpful discussions.

\section{References}

1. Shirts MR, Pitera JW, Swope WC, Pande VS. Extremely precise free energy calculations of amino acid side chain analogs: Comparison of common molecular mechanics force fields for proteins. $\mathrm{J}$ Chem Phys. 2003; 119:5740-5761.

2. Rizzo RC, Aynechi T, Case DA, Kuntz ID. Estimation of absolute free energies of hydration using continuum methods: Accuracy of partial charge models and optimization of nonpolar contributions. J Chem Theory Comput. 2006; 2:128-139. [PubMed: 26626387]

3. Cramer CJ, Truhlar DG. A universal approach to solvation modeling. Acc Chem Res. 2008; 41:7608. [PubMed: 18512970]

4. Klamt A, Mennucci B, Tomasi J, Barone V, Curutchet C, Orozco M, Luque FJ. On the performance of continuum solvation methods . a comment on universal approaches to solvation modeling. Acc Chem Res. 2009; 42:489-492. [PubMed: 19222200]

5. Guthrie JP. A blind challenge for computational solvation free energies : Introduction and overview. J Phys Chem B. 2009; 113:4501-4507. [PubMed: 19338360]

6. Geballe MT, Skillman AG, Nicholls A, Guthrie JP, Taylor PJ. The SAMPL2 blind prediction challenge: introduction and overview. J Comput-Aided Mol Des. 2010; 24:259-79. [PubMed: 20455007]

7. Skillman AG. SAMPL3: blinded prediction of host-guest binding affinities, hydration free energies, and trypsin inhibitors. J Comput-Aided Mol Des. 2012; 26:473-4. [PubMed: 22622621]

8. Geballe MT, Guthrie JP. The SAMPL3 blind prediction challenge: transfer energy overview. J Comput-Aided Mol Des. 2012; 26:489-96. [PubMed: 22476552]

9. Mobley DL, Wymer KL, Lim NM, Guthrie JP. Blind prediction of solvation free energies from the SAMPL4 challenge. J Comput-Aided Mol Des. 2014; 28:135-50. [PubMed: 24615156]

10. König G, Hudson PS, Boresch S, Woodcock HL. Multiscale free energy simulations: An efficient method for connecting classical MD simulations to QM or QM/MM free energies using NonBoltzmann Bennett reweighting schemes. J Chem Theory Comput. 2014; 10:1406-1419. [PubMed: 24803863]

11. König G, Brooks BR. Correcting for bond or angle constraints in free energy simulations during post-processing. Biochim Biophys Acta. 2015; 1850:932-943. [PubMed: 25218695]

12. Genheden S, Cabedo Martinez AI, Criddle MP, Essex JW. Extensive all-atom Monte Carlo sampling and QM/MM corrections in the SAMPL4 hydration free energy challenge. J ComputAided Mol Des. 2014; 28:187-200. [PubMed: 24488307]

13. König G, Pickard FC, Mei Y, Brooks BR. Predicting hydration free energies with a hybrid QM/MM approach: an evaluation of implicit and explicit solvation models in SAMPL4. J ComputAided Mol Des. 2014; 28:245-57. [PubMed: 24504703]

14. König G, Boresch S. Non-Boltzmann sampling and Bennett's acceptance ratio method: How to profit from bending the rules. J Comput Chem. 2011; 32:1082-1090. [PubMed: 21387335]

15. Hu H, Lu Z, Parks JM, Burger SK, Yang W. Quantum mechanics/molecular mechanics minimum free-energy path for accurate reaction energetics in solution and enzymes: sequential sampling and 
optimization on the potential of mean force surface. J Chem Phys. 2008; 128:034105. [PubMed: 18205486]

16. Pulay P, Janowski T. Efficient calculation of the energy of a molecule in an arbitrary electric field. Int J Quantum Chem. 2009; 109:2113-2120.

17. Janowski T, Wolinski K, Pulay P. Ultrafast Quantum Mechanics/Molecular Mechanics Monte Carlo simulations using generalized multipole polarizabilities. Chem Phys Lett. 2012; 530:1-9.

18. Sodt AJ, Mei Y, Koenig G, Tao P, Steele RP, Brooks BR, Shao Y. Multiple Environment Single System Quantum Mechanical/Molecular Mechanical ( MESS-QM/MM ) calculations . 1 . estimation of polarization energies. J Phys Chem A. 2015; 119:1511-1523. [PubMed: 25321186]

19. Marenich AV, Olson RM, Kelly CP, Cramer CJ, Truhlar DG. Self-consistent reaction field model for aqueous and nonaqueous solutions based on accurate polarized partial charges. J Chem Theory Comput. 2007; 3:2011-2033. [PubMed: 26636198]

20. Marenich AV, Cramer CJ, Truhlar DG. Generalized born solvation model SM12. J Chem Theory Comput. 2013; 9:609-620. [PubMed: 26589059]

21. Marenich AV, Cramer CJ, Truhlar DG. Universal solvation model based on solute electron density and on a continuum model of the solvent defined by the bulk dielectric constant and atomic surface tensions. J Phys Chem B. 2009; 113:6378-6396. [PubMed: 19366259]

22. Wolinski K, Pulay P. Second-order Møller-Plesset calculations with dual basis sets. J Chem Phys. 2003; 118:9497.

23. Liang W, Head-Gordon M. Approaching the basis set limit in density functional theory calculations using dual basis sets without diagonalization. J Phys Chem A. 2004; 108:3206-3210.

24. Steele RP, Shao Y, DiStasio RA, Head-Gordon M. Dual-basis analytic gradients. 1. self-consistent field theory. J Phys Chem A. 2006; 110:13915-13922. [PubMed: 17181351]

25. Steele RP, DiStasio RA, Shao Y, Kong J, Head-Gordon M. Dual-basis second-order Møller-Plesset perturbation theory: A reduced-cost reference for correlation calculations. J Chem Phys. 2006; 125:074108. [PubMed: 16942323]

26. Deng J, Gilbert ATB, Gill PMW. Density functional triple jumping. Phys Chem Chem Phys. 2010; 12:10759-10765. [PubMed: 20614077]

27. Brooks BR, Brooks CL, Mackerell AD, Nilsson L, Petrella RJ, Roux B, Won Y, Archontis G, Bartels C, Boresch S, Caflisch A, Caves L, Cui Q, Dinner AR, Feig M, Fischer S, Gao J, Hodoscek M, Im W, Kuczera K, Lazaridis T, Ma J, Ovchinnikov V, Paci E, Pastor RW, Post CB, Pu JZ, Schaefer M, Tidor B, Venable RM, Woodcock HL, Wu X, Yang W, York DM, Karplus M. CHARMM : The biomolecular simulation program. J Comput Chem. 2009; 30:1564-1614.

28. Vanommeslaeghe K, Hatcher E, Acharya C, Kundu S, Zhong S, Shim J, Darian E, Guvench O, Lopes P, Vorobyov I, MacKerell AD. CHARMM general force field : A force field for drug-like molecules compatible with the CHARMM all-atom additive biological force fields. J Comput Chem. 2009; 31:671-690.

29. Vanommeslaeghe K, MacKerell AD. Automation of the CHARMM general force field (CGenFF) I: bond perception and atom typing. J Chem Info Model. 2012; 52:3144-54.

30. Jorgensen WL, Chandrasekhar J, Madura JD, Impey RW, Klein ML. Comparison of simple potential functions for simulating liquid water. J Chem Phys. 1983; 79:926.

31. Mobley DL, Chodera JD, Dill KA. Treating entropy and conformational changes in implicit solvent simulations of small molecules. J Phys Chem B. 2008; 112:938-946. [PubMed: 18171044]

32. Sugita Y, Kitao A, Okamoto Y. Multidimensional replica-exchange method for free-energy calculations. J Chem Phys. 2000; 113:6042.

33. Shao Y, Gan Z, Epifanovsky E, Gilbert AT, Wormit M, Kussmann J, Lange AW, Behn A, Deng J, Feng X, Ghosh D, Goldey M, Horn PR, Jacobson LD, Kaliman I, Khaliullin RZ, Kuś T, Landau A, Liu J, Proynov EI, Rhee YM, Richard RM, Rohrdanz MA, Steele RP, Sundstrom EJ, Woodcock HL, Zimmerman PM, Zuev D, Albrecht B, Alguire E, Austin B, Beran GJO, Bernard YA, Berquist E, Brandhorst K, Bravaya KB, Brown ST, Casanova D, Chang CM, Chen Y, Chien SH, Closser KD, Crittenden DL, Diedenhofen M, DiStasio Ra, Do H, Dutoi AD, Edgar RG, Fatehi S, FustiMolnar L, Ghysels A, Golubeva-Zadorozhnaya A, Gomes J, Hanson-Heine MW, Harbach PH, Hauser AW, Hohenstein EG, Holden ZC, Jagau TC, Ji H, Kaduk B, Khistyaev K, Kim J, Kim J, King RA, Klunzinger P, Kosenkov D, Kowalczyk T, Krauter CM, Lao KU, Laurent A, Lawler KV, 
Levchenko SV, Lin CY, Liu F, Livshits E, Lochan RC, Luenser A, Manohar P, Manzer SF, Mao SP, Mardirossian N, Marenich AV, Maurer SA, Mayhall NJ, Neuscamman E, Oana CM, OlivaresAmaya R, O’Neill DP, Parkhill JA, Perrine TM, Peverati R, Prociuk A, Rehn DR, Rosta E, Russ NJ, Sharada SM, Sharma S, Small DW, Sodt A, Stein T, Stück D, Su YC, Thom AJ, Tsuchimochi T, Vanovschi V, Vogt L, Vydrov O, Wang T, Watson MA, Wenzel J, White A, Williams CF, Yang J, Yeganeh S, Yost SR, You ZQ, Zhang IY, Zhang X, Zhao Y, Brooks BR, Chan GK, Chipman DM, Cramer CJ, Goddard WA, Gordon MS, Hehre WJ, Klamt A, Schaefer HF, Schmidt MW, Sherrill CD, Truhlar DG, Warshel A, Xu X, Aspuru-Guzik A, Baer R, Bell AT, Besley NA, Chai JD, Dreuw A, Dunietz BD, Furlani TR, Gwaltney SR, Hsu CP, Jung Y, Kong J, Lambrecht DS, Liang W, Ochsenfeld C, Rassolov VA, Slipchenko LV, Subotnik JE, Van Voorhis T, Herbert JM, Krylov AI, Gill PM, Head-Gordon M. Advances in molecular quantum chemistry contained in the q-chem 4 program package. Mol Phys. 2015; 113:184-215.

34. Woodcock HL, Hodošček M, Gilbert ATB, Gill PMW, Schaefer HF, Brooks BR. Interfacing QChem and CHARMM to perform QM/MM reaction path calculations. J Comput Chem. 2007; 28:1485-1502. [PubMed: 17334987]

35. Chai JD, Head-Gordon M. Long-range corrected hybrid density functionals with damped atomatom dispersion corrections. Phys Chem Chem Phys. 2008; 10:6615-6620. [PubMed: 18989472]

36. Becke AD. Density-functional exchange-energy approximation with correct asymptotic behavior. Phys Rev A. 1988; 38:3098-3100.

37. Lee C, Yang W, Parr RG. Development of the Colle-Salvetti correlation-energy formula into a functional of the electron density. Phys Rev B. 1988; 37:785-789.

38. Becke AD. A new mixing of Hartree-Fock and local density functional theories. J Chem Phys. 1993; 98:1372.

39. Adamo C, Barone V. Toward reliable density functional methods without adjustable parameters: The PBE0 model. J Chem Phys. 1999; 110:6158.

40. Zhao Y, Truhlar DG. The M06 suite of density functionals for main group thermochemistry, thermochemical kinetics, noncovalent interactions, excited states, and transition elements: two new functionals and systematic testing of four M06-class functionals and 12 other function. Theor Chem Acc. 2007; 120:215-241.

41. Becke AD. A multicenter numerical integration scheme for polyatomic molecules. J Chem Phys. 1988; 88:2547.

42. Goldey M, Head-Gordon M. Attenuating away the errors in inter- and intramolecular interactions from second-order Møller-Plesset calculations in the small aug-cc-pVDZ basis set. J Phys Chem Lett. 2012; 3:3592-3598. [PubMed: 26290993]

43. Goldey M, Dutoi A, Head-Gordon M. Attenuated second-order Møller-Plesset perturbation theory: performance within the aug-cc-pVTZ basis. Phys Chem Chem Phys. 2013; 15:15869-75. [PubMed: 23942866]

44. Zhang IY, Xu X, Jung Y, Goddard WA. A fast doubly hybrid density functional method close to chemical accuracy using a local opposite spin ansatz. Proc Nat Acad Sci USA. 2011; 108:19896900. [PubMed: 22114187]

45. Ji H, Shao Y, Goddard WA, Jung Y. Analytic derivatives of quartic-scaling doubly hybrid XYGJOS functional: Theory, implementation, and benchmark comparison with M06-2X and MP2 geometries for nonbonded complexes. J Chem Theory Comput. 2013130319160751008.

46. Olson RM, Marenich AV, Cramer CJ, Truhlar DG. Charge model 4 and intramolecular charge polarization. J Chem Theory Comput. 2007; 3:2046-2054. [PubMed: 26636200]

47. Singh UC, Kollman PA. An approach to computing electrostatic charges for molecules. J Comput Chem. 1984; 5:129-145.

48. Besler BH, Merz KM, Kollman PA. Atomic charges derived from semiempirical methods. J Comput Chem. 1990; 11:431-439.

49. Breneman CM, Wiberg KB. Determining atom-centered monopoles from molecular electrostatic potentials. the need for high sampling density in formamide conformational analysis. J Comput Chem. 1990; 11:361-373. 
50. Herbert JM, Jacobson LD, Lao KU, Rohrdanz MA. Rapid computation of intermolecular interactions in molecular and ionic clusters: self-consistent polarization plus symmetry-adapted perturbation theory. Phys Chem Chem Phys. 2012; 14:7679-99. [PubMed: 22511183]

51. Holden ZC, Richard RM, Herbert JM. Periodic boundary conditions for QM/MM calculations: Ewald summation for extended gaussian basis sets. J Chem Phys. 2013; 139:244108. [PubMed: 24387358]

52. Guthrie JP. SAMPL4: a blind challenge for computational solvation free energies: the compounds considered. J Comput-Aided Mol Des. 2014; 28:151-68. [PubMed: 24706106]

53. Tkatchenko A, DiStasio RA, Car R, Scheffer M. Accurate and efficient method for many-body van der Waals interactions. Phys Rev Lett. 2012; 108:236402. [PubMed: 23003978]

54. DiStasio RA, Gobre VV, Tkatchenko A. Many-body van der Waals interactions in molecules and condensed matter. J Phys Condens Matt. 2014; 26:213202.

55. Shaw KE, Woods CJ, Mulholland AJ. Compatibility of quantum chemical methods and empirical (MM) water models in Quantum Mechanics/Molecular Mechanics liquid water simulations. J Phys Chem Lett. 2010; 1:219-223.

56. Wolfenden R, Andersson L, Cullis PM, Southgate CC. Affinities of amino acid side chains for solvent water. Biochemistry. 1981; 20:849-855. [PubMed: 7213619]

57. Ben-Naim A, Marcus Y. Solvation thermodynamics of nonionic solutes. J Chem Phys. 1984; 81:2016.

58. Straatsma TP, McCammon JA. Treatment of rotational isomers in free energy evaluations. analysis of the evaluation of free energy differences by molecular dynamics simulations of systems with rotational isomeric states. J Chem Phys. 1989; 90:3300-3304.

59. Tao P, Sodt AJ, Shao Y, König G, Brooks BR. Computing the free energy along a reaction coordinate using rigid body dynamics. J Chem Theory Comput. 2014; 10:4198-4207. [PubMed: 25328492]

60. Kästner J, Thiel S, Senn HM, Sherwood P, Thiel W. Exploiting QM/MM capabilities in geometry optimization: A microiterative approach using electrostatic embedding. J Chem Theory Comput. 2007; 3:1064-1072. [PubMed: 26627425]

61. Ram CL, Zeida A, Jara GE, Roitberg E, Mart MA. Improving efficiency in SMD simulations through a hybrid differential relaxation algorithm. J Chem Theory Comput. 2014; 10:4609-4617. [PubMed: 26588154]

62. Babin V, Leforestier C, Paesani F. Development of a "first principles water potential with flexible monomers: Dimer potential energy surface, VRT spectrum, and second virial coefficient". J Chem Theory Comput. 2013; 9:5395-5403. [PubMed: 26592277]

63. Babin V, Medders GR, Paesani F. Development of a "first principles water potential with flexible monomers II: Trimer potential energy surface, third virial coefficient, and small clusters". J Chem Theory Comput. 2014; 10:1599-1607. [PubMed: 26580372]

64. Medders GR, Babin V, Paesani F. Development of a "first principles water potential with flexible monomers III: Liquid phase properties". J Chem Theory Comput. 2014; 10:2906-2910. [PubMed: 26588266]

65. Ischtwan J, Collins M. Molecular potential energy surfaces by interpolation. J Chem Phys. 1994; 100:8080-8088.

66. Park JW, Rhee YM. Interpolated mechanics-molecular mechanics study of internal rotation dynamics of the chromophore unit in blue fluorescent protein and its variants. J Phys Chem B. 2012; 116:11137-47. [PubMed: 22891786] 

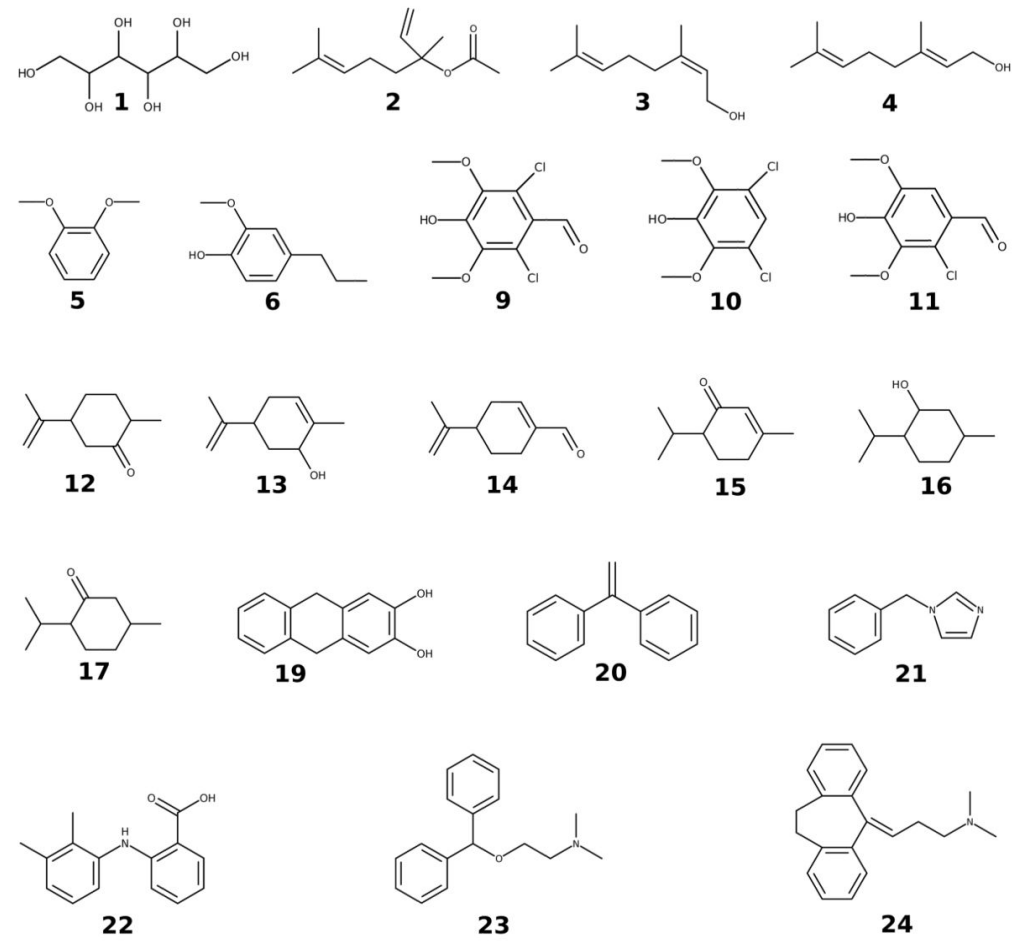

23

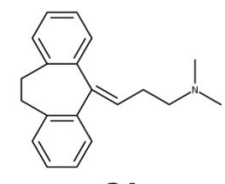

(a)
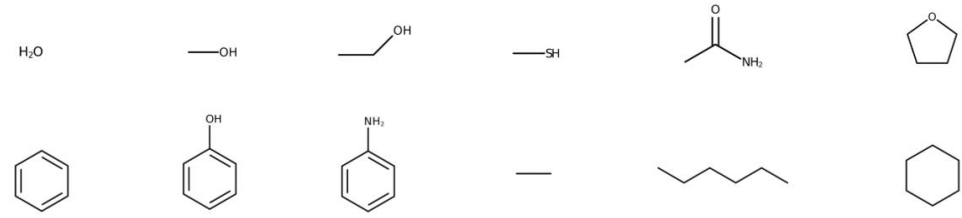

(b)

FIG. 1.

(a) SAMPL4 molecules and (b) twelve small molecules studied in this work. 


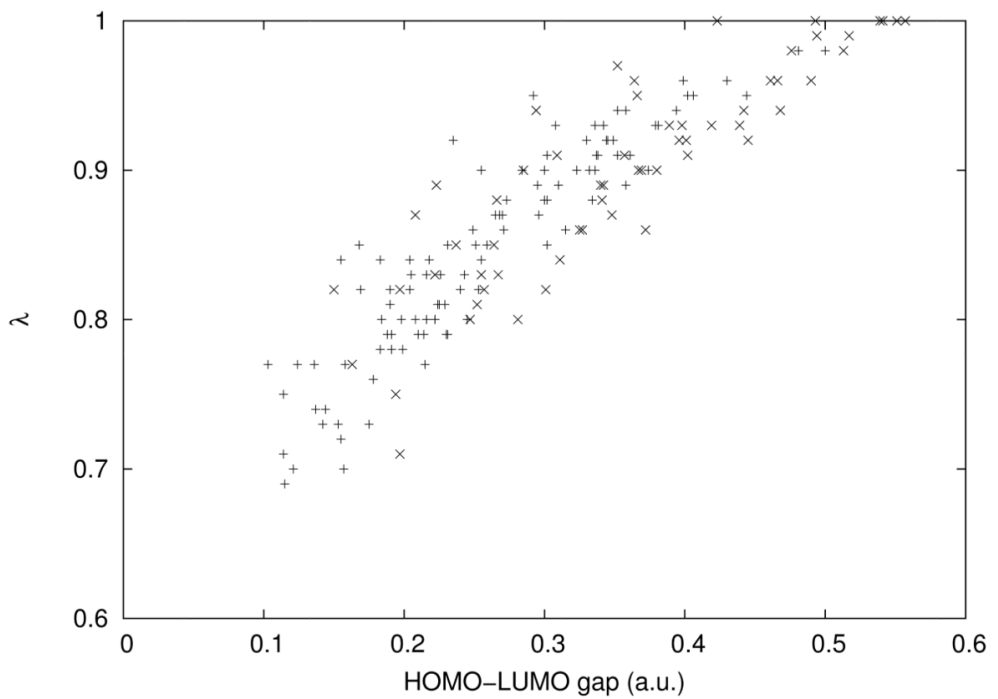

FIG. 2.

Computed $\lambda$ values vs HOMO-LUMO gap for SAMPL4 molecules (denoted with “+”) and twelve small molecules (denoted by " $x$ ”). 


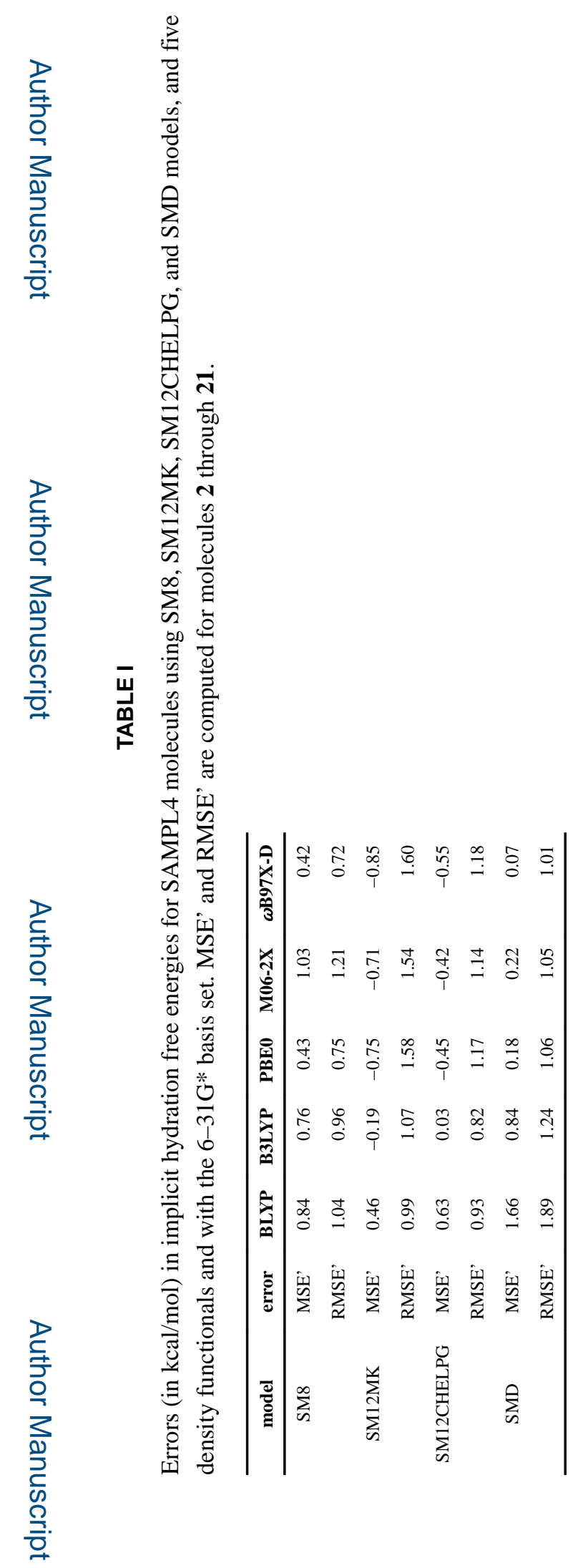




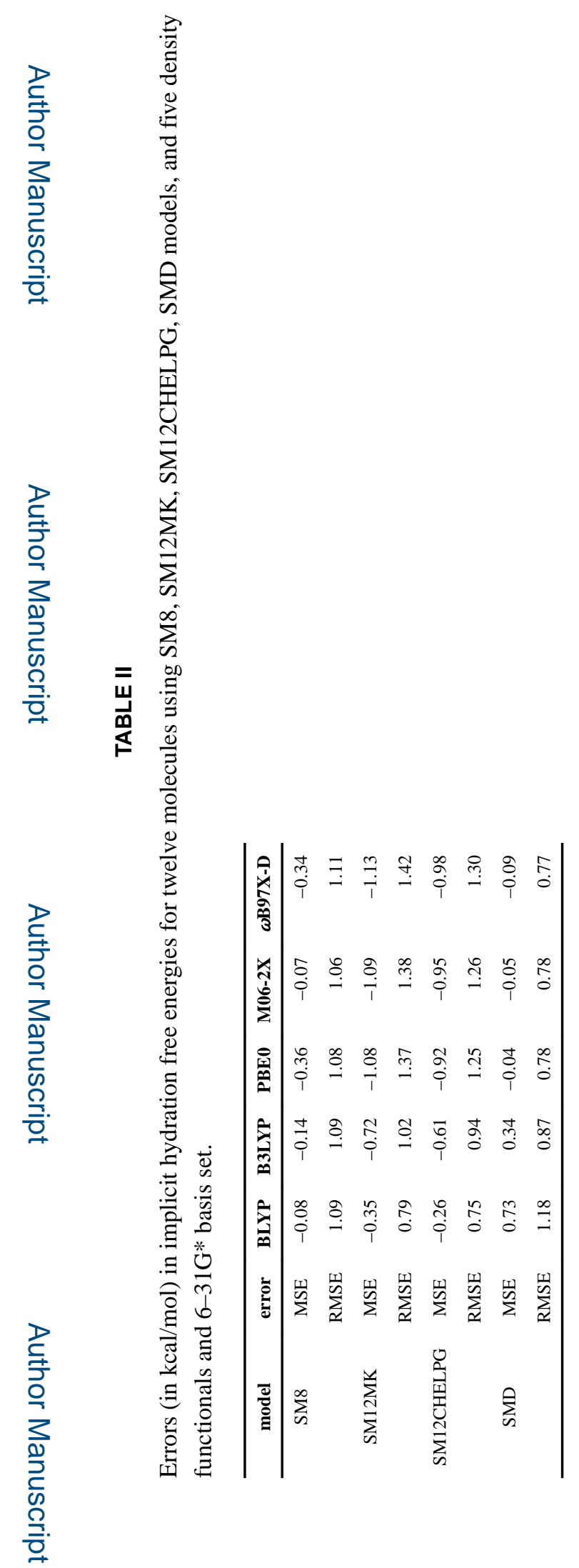


TABLE III

Solvation free energies in kcal/mol from QM/MM-NBB and MESS-E-QM/MM NBB calculations using M06-2X/6-31G*. MSE' and RMSE' are computed for molecules 2 through 21, against full QM/MM-NBB results (column 2).

\begin{tabular}{|c|c|c|}
\hline molecule & QM/MM-NBB & MESS-E-QM/MM-NBB \\
\hline 1 & $-22.22 \pm 0.44$ & $-22.04 \pm 0.42$ \\
\hline 2 & $-4.18 \pm 0.10$ & $-4.27 \pm 0.13$ \\
\hline 3 & $-7.52 \pm 0.07$ & $-7.47 \pm 0.08$ \\
\hline 4 & $-7.21 \pm 0.23$ & $-7.09 \pm 0.25$ \\
\hline 5 & $-6.76 \pm 0.53$ & $-6.51 \pm 0.46$ \\
\hline 6 & $-10.87 \pm 0.62$ & $-10.37 \pm 0.51$ \\
\hline 9 & $-10.57 \pm 0.98$ & $-10.27 \pm 0.91$ \\
\hline 10 & $-6.85 \pm 0.20$ & $-6.70 \pm 0.19$ \\
\hline 11 & $-9.23 \pm 0.37$ & $-9.10 \pm 0.38$ \\
\hline 12 & $-5.31 \pm 0.19$ & $-5.25 \pm 0.20$ \\
\hline 13 & $-6.63 \pm 0.21$ & $-6.52 \pm 0.21$ \\
\hline 14 & $-5.15 \pm 0.30$ & $-5.29 \pm 0.36$ \\
\hline 15 & $-6.45 \pm 0.25$ & $-6.53 \pm 0.30$ \\
\hline 16 & $-5.04 \pm 0.30$ & $-5.07 \pm 0.30$ \\
\hline 17 & $-4.34 \pm 0.19$ & $-4.49 \pm 0.21$ \\
\hline 19 & $-5.23 \pm 0.23$ & $-5.05 \pm 0.21$ \\
\hline 20 & $-3.70 \pm 0.29$ & $-3.64 \pm 0.30$ \\
\hline 21 & $-10.04 \pm 0.32$ & $-9.95 \pm 0.38$ \\
\hline 22 & $-10.19 \pm 0.20$ & $-10.09 \pm 0.18$ \\
\hline 23 & $-6.39 \pm 0.27$ & $-6.33 \pm 0.27$ \\
\hline 24 & $-7.35 \pm 0.25$ & $-7.48 \pm 0.28$ \\
\hline MSE' & & 0.09 \\
\hline RMSE' & & 0.18 \\
\hline
\end{tabular}




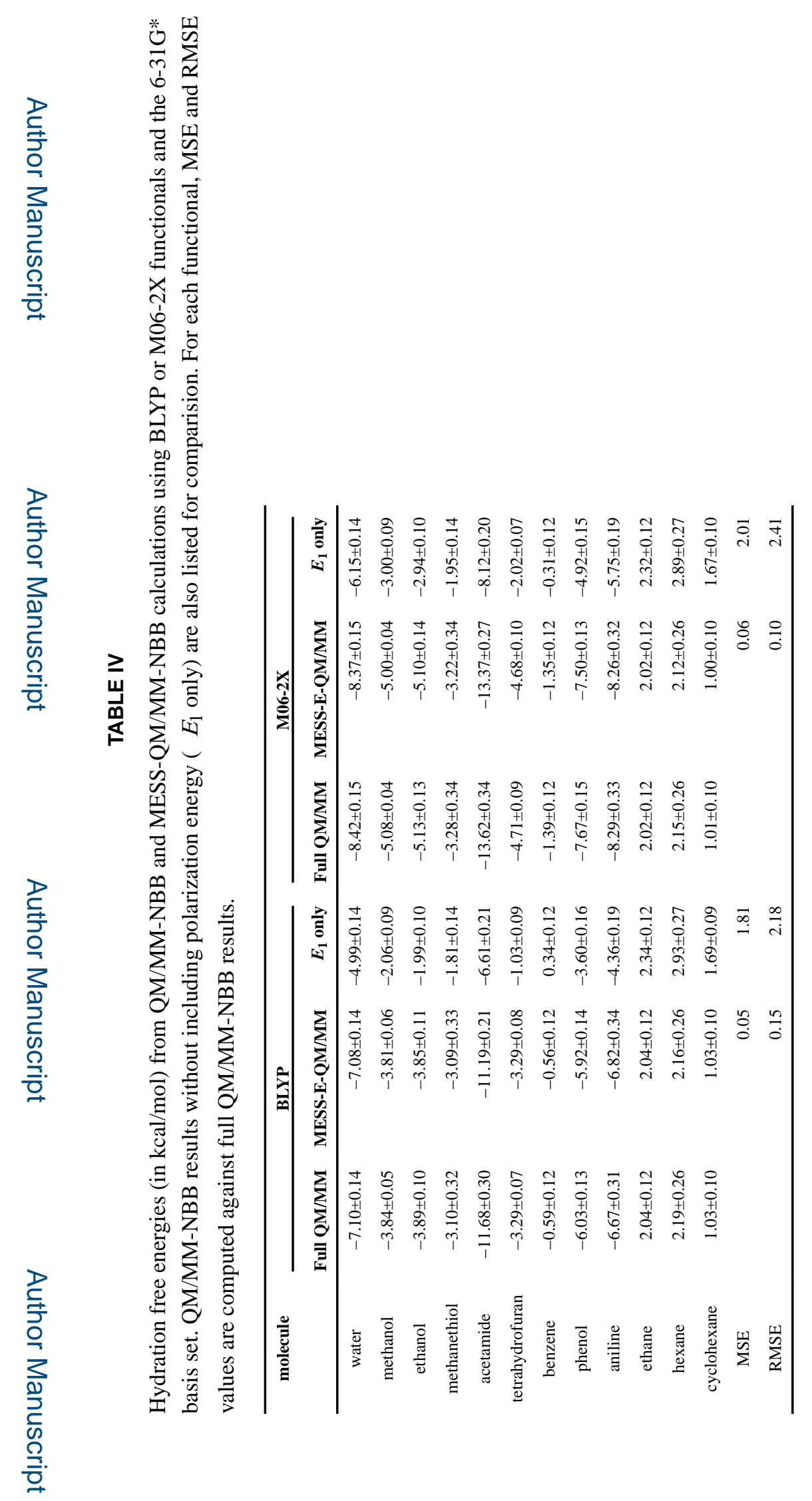

J Chem Theory Comput. Author manuscript; available in PMC 2017 January 25. 


\section{TABLE V}

Computational time for hydration free energy calculations using a single core of an Intel Xeon E5520 2.27GHz processor. For $\mathrm{QM} / \mathrm{MM}$ corrections, M06-2X calculations were performed using the $6-31 \mathrm{G}^{*}$ basis set and a $(99,590)$ grid.

\begin{tabular}{ll}
\hline & Time (CPU hours ) \\
\hline MM & 2,500 \\
Full QM/MM-NBB correction & $10,000-65,000$ \\
MESS-E-QM/MM-NBB correction & $160-300$ \\
\hline
\end{tabular}


König et al.

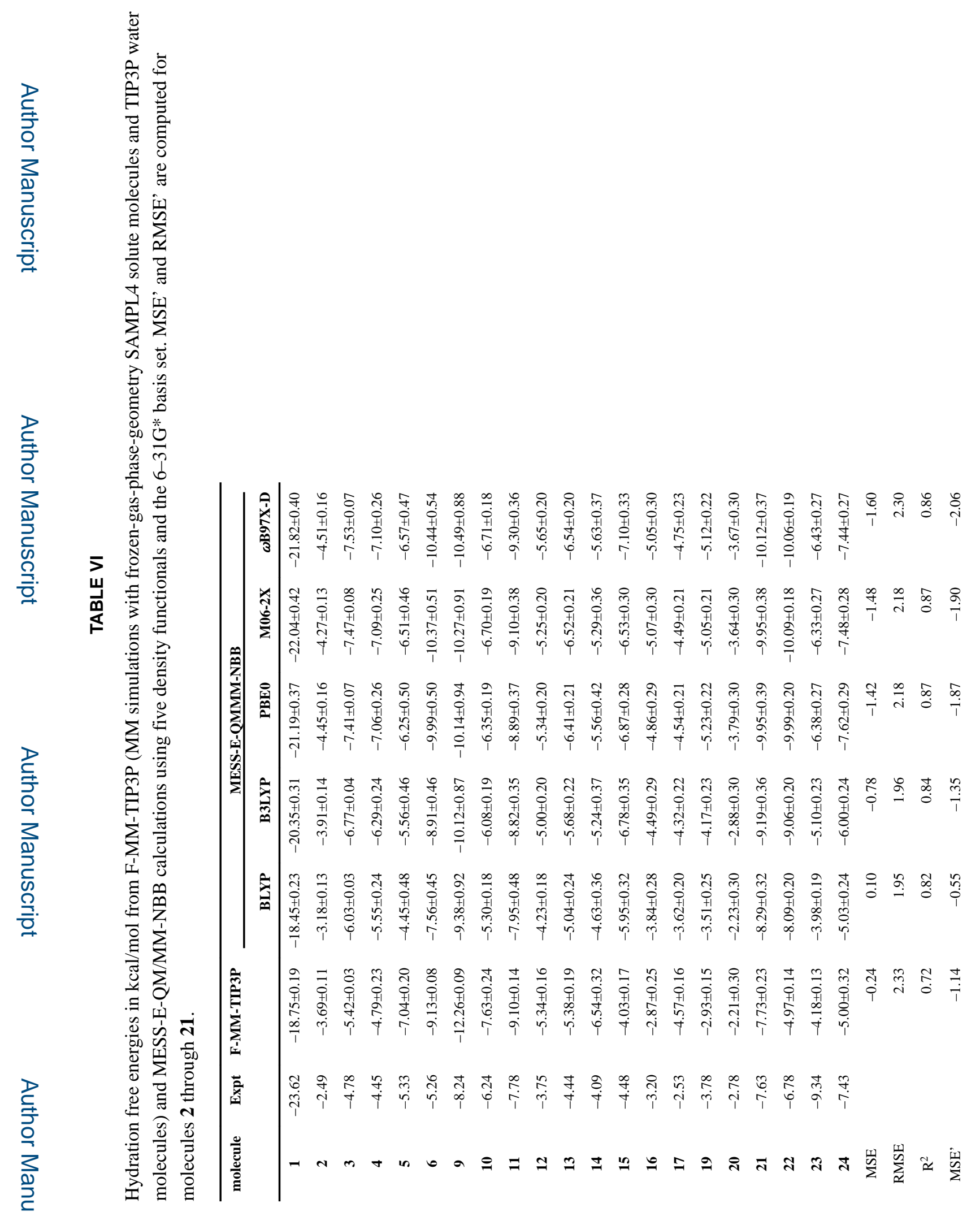

J Chem Theory Comput. Author manuscript; available in PMC 2017 January 25. 


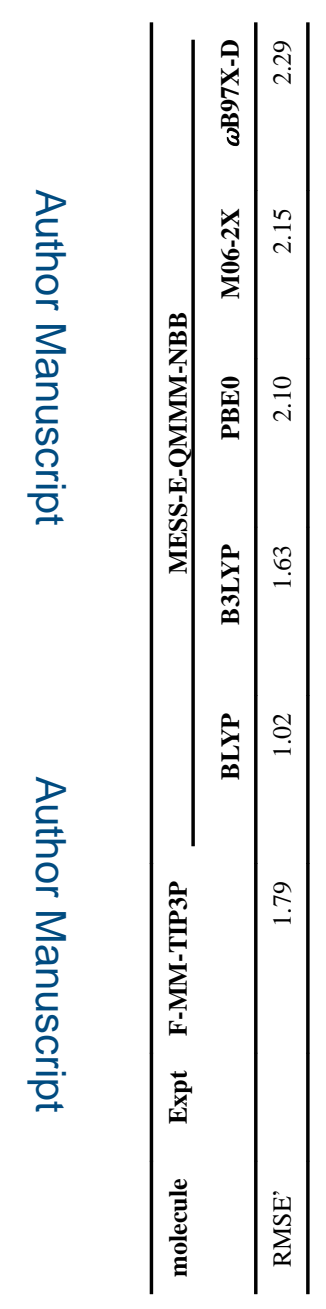

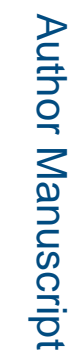

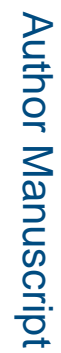




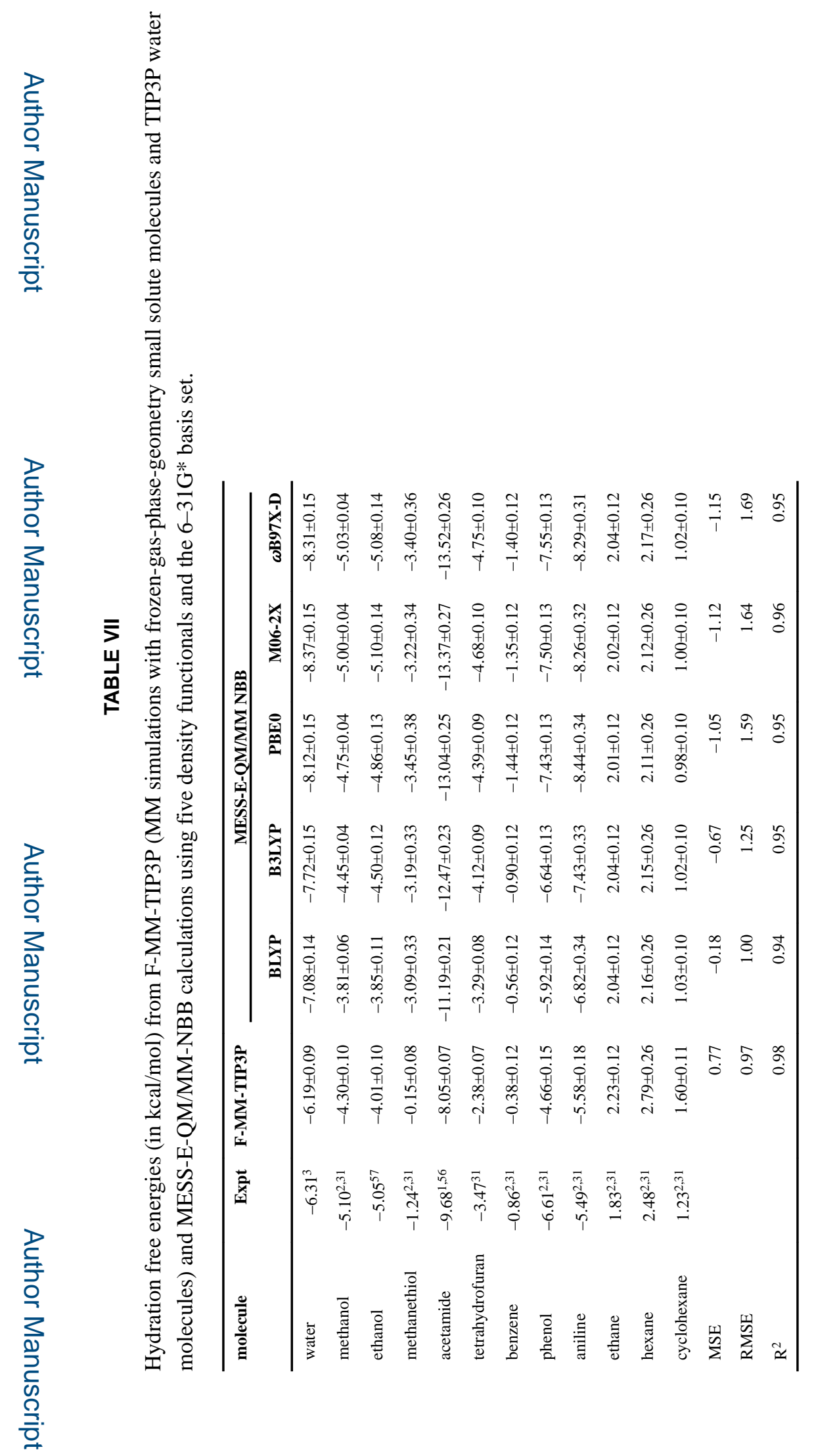

J Chem Theory Comput. Author manuscript; available in PMC 2017 January 25. 
TABLE VIII

Hydration free energies (in kcal/mol) from QM/MM-NBB calculations using ATT-RIMP2/aug-cc-pVDZ or XYGJ-OS/6-311+G(3df,2p) methods for the solute molecules.

\begin{tabular}{crrr}
\hline molecule & \multirow{2}{*}{ Expt } & \multicolumn{2}{c}{ QMMM-NBB } \\
\cline { 3 - 4 } & & ATT-RIMP2 & \multicolumn{1}{c}{ XYGJ-OS } \\
\hline water & -6.31 & $-6.85 \pm 0.14$ & $-7.50 \pm 0.15$ \\
methanol & -5.10 & $-4.81 \pm 0.06$ & $-5.23 \pm 0.06$ \\
ethanol & -5.05 & $-5.18 \pm 0.15$ & $-5.48 \pm 0.16$ \\
methanethiol & -1.24 & $-3.48 \pm 0.37$ & $-3.10 \pm 0.38$ \\
acetamide & -9.68 & $-14.31 \pm 0.57$ & $-15.02 \pm 0.65$ \\
tetrahydrofuran & -3.47 & $-4.99 \pm 0.13$ & $-5.25 \pm 0.14$ \\
benzene & -0.86 & $-1.48 \pm 0.12$ & $-1.85 \pm 0.12$ \\
phenol & -6.61 & $-7.77 \pm 0.11$ & $-8.26 \pm 0.14$ \\
aniline & -5.49 & $-8.20 \pm 0.30$ & $-8.66 \pm 0.32$ \\
ethane & 1.83 & $1.87 \pm 0.12$ & $1.90 \pm 0.12$ \\
hexane & 2.48 & $1.80 \pm 0.26$ & $1.88 \pm 0.25$ \\
cyclohexane & 1.23 & $0.75 \pm 0.11$ & $0.81 \pm 0.11$ \\
MSE & & -1.20 & -1.46 \\
RMSE & & 1.80 & 2.06 \\
$\mathrm{R}^{2}$ & & 0.93 & 0.95 \\
\hline
\end{tabular}

